DESY 07-074

NSF-KITP-07-128

KCL-MTH-07-06

arXive/0706.0744

\title{
Free fermion resolution of supergroup WZNW models
}

\author{
Thomas Quella ${ }^{1}$ and Volker Schomerus ${ }^{2}$ \\ ${ }^{1}$ Korteweg-de Vries Institute for Mathematics, University of Amsterdam, \\ Plantage Muidergracht 24, 1018 TV Amsterdam, The Netherlands \\ 2 DESY Theory Group, DESY Hamburg, \\ Notkestrasse 85, D-22603 Hamburg, Germany
}

\begin{abstract}
Extending our earlier work on $P S L(2 \mid 2)$, we explain how to reduce the solution of WZNW models on general type I supergroups to those defined on the bosonic subgroup. The new analysis covers in particular the supergroups $G L(M \mid N)$ along with several close relatives such as $P S L(N \mid N)$, certain Poincaré supergroups and the series $O S P(2 \mid 2 N)$. This remarkable progress relies on the use of a special Feigin-Fuchs type representation. In preparation for the field theory analysis, we shall exploit a minisuperspace analogue of a free fermion construction to deduce the spectrum of the Laplacian on type I supergroups. The latter is shown to be non-diagonalizable. After lifting these results to the full WZNW model, we address various issues of the field theory, including its modular invariance and the computation of correlation functions. In agreement with previous findings, supergroup WZNW models allow to study chiral and non-chiral aspects of logarithmic conformal field theory within a geometric framework. We shall briefly indicate how insights from WZNW models carry over to non-geometric examples, such as e.g. the $\mathcal{W}(p)$ triplet models.
\end{abstract}

Keywords: Conformal Field Theory, Logarithmic Conformal Field Theory, Free Field Constructions, Supergroups, Lie Superalgebras, Representation Theory 


\section{Contents}

1 Introduction $\quad 2$

2 Some background on Lie superalgebras of type I 5

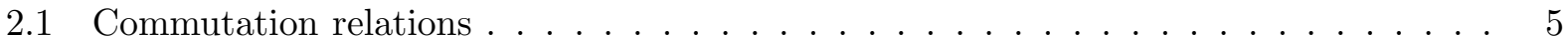

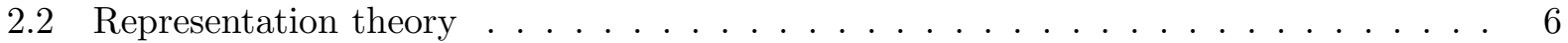

2.2.1 Kac modules and their duals . . . . . . . . . . . . . . 7

2.2 .2 Simple modules and their blocks . . . . . . . . . . . . . . 8

2.2 .3 Projective modules . . . . . . . . . . . . . . . . . . . . 9

2.2.4 The quadratic Casimir element . . . . . . . . . . . . . . 11

3 The supergroup WZNW model and its symmetries 11

3.1 The Lagrangian description . . . . . . . . . . . . . . . . . . . 12

3.2 Covariant formulation of the symmetry . . . . . . . . . . . . 14

3.3 Free fermion resolution . . . . . . . . . . . . . . . . . . 16

4 Semi-classical analysis $\quad 17$

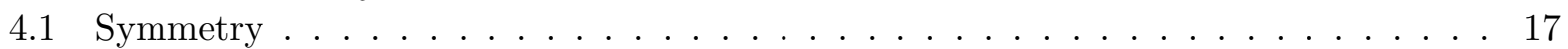

4.2 Harmonic analysis . . . . . . . . . . . . . . . . . . . . . . 19

4.3 Spectrum and generalized eigenfunctions . . . . . . . . . . . . . . . . . 20

4.4 Correlation functions . . . . . . . . . . . . . . . . . 23

5 The quantum WZNW model 2

5.1 Chiral representations of the current superalgebra $\ldots \ldots \ldots \ldots$

5.2 Spectral flow automorphisms . . . . . . . . . . . . . . . 28

5.3 Spectrum and correlation functions . . . . . . . . . . . . . . 30

5.4 Some comments on non-trivial modular invariants . . . . . . . . . . . . . 34

6 Lessons for other logarithmic CFTs

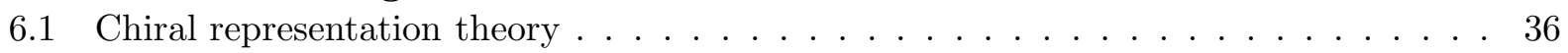

6.2 Local logarithmic conformal field theories . . . . . . . . . . . . . 37

$\begin{array}{lll}7 & \text { Outlook and open questions } & 39\end{array}$

\section{Introduction}

Two-dimensional non-linear $\sigma$-models on supermanifolds have been a topic of considerable interest for the past few decades. Their realm of applications is vast, ranging from string theory to statistical physics and condensed matter theory. In the Green-Schwarz or pure spinor type formulation of superstring theory, for example, supersymmetries act geometrically as isometries of an underlying space-time (target space) supermanifold. Important examples arise in the context of AdS/CFT dualities between supersymmetric gauge theories and closed strings. Apart from string theory, supersymmetry has also played a major role in the context of quantum disordered systems [1, 2, 3, 4] and in models with non-local degrees of freedom such as polymers [5]. In particular, it seems to be a crucial ingredient in the description of the plateaux transitions in the spin [6, 7] and the integer quantum Hall effect [8, 9, 10]. 
In addition to such concrete applications there exist a number of structural reasons to be interested in conformal $\sigma$-models with target space (internal) supersymmetry. On the one hand, being non-unitary, the relevant conformal field theory models exhibit rather unusual features such as the occurrence of reducible but indecomposable 1 representations and the existence of logarithmic singularities on the world-sheet. In this context, many conceptual issues remain to be solved, both on the physical and on the mathematical side. These include, in particular, the construction of consistent local correlation functions [11], the modular transformation properties of characters [12, 13], their relation to fusion rules [14, 15, 16], the treatment of conformal boundary conditions [17, 18] etc. On the other hand, the special properties of Lie supergroups allow for constructions which are not possible for ordinary groups. For instance, there exist several families of coset conformal field theories that are obtained by gauging a one-sided action of some subgroup rather than the usual adjoint [19, 20, 21, 22]. The same class of supergroup $\sigma$-models is also known to admit a new kind of marginal deformations that are not of current-current type [23, 24]. Finally, there seems to be a striking correspondence between the integrability of these models and their conformal invariance [25, 26, 21, 22,

In this note we will focus on the simplest class of two-dimensional conformal $\sigma$-models, namely WZNW theories, in order to address some of the features mentioned above. The two essential properties which facilitate an exact solution are (i) the presence of an extended chiral symmetry based on an infinite dimensional current superalgebra 2 and (ii) the inherent geometric interpretation. While (ii) is common to all $\sigma$-models, the symmetries of WZNW models are necessary to lift geometric insights to the full field theory. Both aspects single out supergroup WZNW theories among most of the logarithmic conformal field theories that have been considered in the past [27, 11, 28, (see also [29, 30] for reviews and further references). While investigations of algebraic and mostly chiral aspects of supergroup WZNW models reach back more than ten years [31, 32, 33, 34, 35] it was not until recently that the use of geometric methods has substantially furthered our understanding of non-chiral issues [36, 24, 37]. In the last three references the full non-chiral spectrum for the $G L(1 \mid 1)$, the $P S U(1,1 \mid 2)$ and the $S U(2 \mid 1)$ WZNW models has been derived based on methods of harmonic analysis. The most important discovery in these articles was the relevance of socalled projective covers and the resulting non-diagonalizability of the Laplacian which ultimately manifests itself in the logarithmic behaviour of correlation functions.

This paper will put these results on a more general and firm conceptual basis by considering rather arbitrary supergroup WZNW models based on basic Lie superalgebras of type I. The defining properties of these Lie superalgebras are (i) the existence of a non-degenerate invariant form (not necessarily the Killing form) and (ii) the possibility to split the fermionic generators into two multiplets which transform in dual representations of the even subalgebra. The first feature is necessary to even spell out a Lagrangian for our models. Our second requirement can be exploited to introduce a distinguished set of coordinates in which the Lagrangian takes a particularly simple form. These arise from some Gauss-like decomposition in which a bosonic group element is sandwiched between the two sets of fermions. The construction resembles the free field construction of bosonic models [38, 39, 40, 41, 42], but its fermionic version turns out to be easier to deal with

\footnotetext{
${ }^{1}$ In contrast to some appearances in the physics literature we will use the word "indecomposable" strictly in the mathematical sense. According to that definition also irreducible representations are always indecomposable since they cannot be written as a direct sum of two other (non-zero) representations.

${ }^{2}$ Instead of referring to the names "Kac-Moody superalgebra" or even "affine Lie superalgebra" which are frequently used in the physics community, we will stick to the notion current superalgebra by which we mean a central extension of the loop algebra over a finite dimensional Lie superalgebra.
} 
since the corresponding Gauss decomposition is globally defined. We shall make no specific choice concerning the coordinates on the bosonic subgroup so that the underlying bosonic symmetry is manifest throughout our construction 3 The generators of the underlying current superalgebra of our WZNW model are thus constructed from currents of the bosonic subalgebra along with a number of free chiral fermionic ghost systems which equals the number of fermionic generators.

As was observed in [24] already, at least for the example of $\operatorname{PSU}(1,1 \mid 2)$, the free fermion resolution described above provides a natural framework for the discussion of representations, spectrum, characters and correlation functions, both in the full conformal field theory and in its semi-classical subsector. In particular, it is possible to introduce the notion of "Kac modules" for current superalgebras. These are obtained as a tensor product of an irreducible highest weight representation of the (renormalized) bosonic subalgebra and a Fock space for the free fermions. Exactly as their finite dimensional cousins, such Kac modules turn out to be irreducible for generic (typical) choices of the highest weight. Hence, their characters can be written down immediately and their behaviour under modular transformations is straightforward to derive. Though our presentation does not allow to elaborate on the details without specifying a concrete model, we believe that techniques similar to the ones used in [32, 24] also permit to derive characters for atypical irreducible representations. The latter arise as quotients from reducible Kac modules and their characters possess representations through infinite sums over characters of Kac modules. Such formulas were shown in [32, 37] to provide easy access to modular properties of atypical irreducibles.

If we restrict our attention to simple Lie superalgebras for a moment our analysis covers three types of infinite series, namely $A(m, n)=\operatorname{sl}(m \mid n)$ (for $m \neq n), A(n, n)=\operatorname{psl}(n \mid n)$ and $C(n+$ $1)=\operatorname{osp}(2 \mid 2 n)$ [45]. But, widening Kac's original usage of the qualifiers "basic" and "type I", most of our results also apply to non-(semi)simple Lie superalgebras such as various extended Poincaré superalgebras, the general linear Lie superalgebras $g l(m \mid n)$ or supersymmetric extensions of Heisenberg algebras 4 We wish to stress that our general results below contain a solution of the $P S L(n \mid n)$ WZNW models. What makes these particularly interesting is the fact that their volume is an exact modulus, in contrast to bosonic non-abelian WZNW models [23, 22]. It is also worth emphasizing that the isometries of flat superspace, AdS-spaces and many projective superspaces fall into the classes mentioned above. We thus expect our work to be relevant for these models as well. A few comments in that direction can be found in the conclusions.

The plan of this paper is as follows. In the next section we shall provide a detailed account of Lie superalgebras of type I and the associated representation theory. Particular emphasis is put on the structure of projective modules, i.e. typical Kac modules and projective covers of atypical irreducible representations. Afterwards we present the supergroup WZNW Lagrangian in section 3 and use a Gauss-like decomposition in order to rewrite it in terms of a bosonic WZNW model, two sets of free fermions and an interaction term which couples bosons and fermions. This free fermion resolution is shown to have an algebraic analogue on the level of the current superalgebra which constitutes the symmetry of the supergroup WZNW model. The analysis of the zero-mode spectrum in the large volume sector is performed in section 4 using methods of harmonic analysis. Most importantly, we shall determine the representation content for the combined left right regular action on the algebra of functions over the supergroup. To achieve our goal, we use a reciprocity between atypical irreducible representations and their projective covers. On the way we also prove

\footnotetext{
${ }^{3}$ See 43,44 for a related approach.

${ }^{4}$ WZNW models based on Heisenberg algebras may be used to describe strings on maximally symmetric plane waves [46, 47].
} 
the occurrence of a sector on which the Laplacian is not diagonalizable. After these preparations we extend the free fermion resolution to the full WZNW model in section 5. We introduce an analogue of Kac modules suitable for infinite dimensional Lie superalgebras and sketch the calculation of correlation functions. The latter are necessarily logarithmic due to the non-diagonalizability of the dilatation operators $L_{0}$ and $\bar{L}_{0}$. At the end of section [5, we propose a universal partition function resembling a charge conjugate invariant and gather some thoughts about the possibility of having non-trivial modular invariant partition functions. In the concluding section 6 we argue that the solution of the logarithmic triplet model [11] formally fits into the framework outlined before. This observation is used to speculate about the structure of general logarithmic conformal field theories.

Most of the statements which appear in the main text can be turned into mathematically rigorous propositions. This applies in particular to all algebraic manipulations. In our discussion of spectra, however, we focus on models based on finite dimensional representations. The most interesting supergroups, on the other hand, are based on non-compact and occasionally on nonreducive groups. While we believe that our discussion may be extended to such cases, a fully comprehensive presentation would have required to carefully distinguish between different real forms. In the present note, we rather preferred to put the emphasis on the algebraic structures that - in our opinion - are equally relevant for all type I supergroup WZNW models.

\section{Some background on Lie superalgebras of type I}

The main actress of this paper, the Lie supergroup $G$, is best introduced in terms of its underlying Lie superalgebra $\mathfrak{g}$. We will assume the latter to be finite dimensional, basic and of type I. The attribute "basic" guarantees the existence of a non-degenerate invariant metric and is needed in order to exclude certain pathological cases which would even rule out the existence of a WZNW Lagrangian. The predicate "type I", on the other hand, implies the existence of two multiplets of fermionic generators and will simplify the interpretation of the chiral splitting in the conformal field theory we are considering.

In the remainder of this section we shall first present the commutation relations of a general (possibly non-simple) basic Lie superalgebra of type I. Afterwards we summarize their representation theory following the beautiful exposition of Zou [48] (see also [49]). The reader who is not interested in the mathematical details might wish to skip over parts of this section in the first reading.

\subsection{Commutation relations}

A Lie superalgebra $\mathfrak{g}=\mathfrak{g}_{0} \oplus \mathfrak{g}_{1}$ is a graded generalization of an ordinary Lie algebra [45]. There are even (or bosonic) generators $K^{i}$ which form an ordinary Lie algebra $\mathfrak{g}_{0}$, i.e. they obey the commutation relations

$$
\left[K^{i}, K^{j}\right]=i f^{i j}{ }_{l} K^{l}
$$

with structure constants that are antisymmetric in the upper indices and that satisfy the Jacobi identity. In addition, type I Lie superalgebras possess two sets of odd (or fermionic) generators $S_{1}^{a}$ and $S_{2 a}, a=1, \ldots, r$ (generating $\mathfrak{g}_{1}$ ) which transform in an $r$-dimensional representation $R$ of $\mathfrak{g}_{0}$ and its dual $R^{*}$, respectively [50]. Rephrased in terms of commutation relations, this statement may be expressed as

$$
\left[K^{i}, S_{1}^{a}\right]=-\left(R^{i}\right)^{a}{ }_{b} S_{1}^{b} \quad\left[K^{i}, S_{2 a}\right]=S_{2 b}\left(R^{i}\right)^{b}{ }_{a} .
$$


The symbol $R^{i}$ is an abbreviation for the representation matrix $R\left(K^{i}\right)$. In a type I superalgebra, the anti-commutators $\left[S_{1}^{a}, S_{1}^{b}\right]$ and $\left[S_{2 a}, S_{2 b}\right]$ vanish identically [50]. On the other hand, generators $S_{1}^{a}$ do not anti-commute with $S_{2 b}$. Before we are able to spell out their commutation relations, we need to introduce the supersymmetric bilinear form

$$
\left\langle K^{i}, K^{j}\right\rangle=\kappa^{i j} \quad\left\langle S_{1}^{a}, S_{2 b}\right\rangle=\delta_{b}^{a} .
$$

We assume $\kappa^{i j}$ (not necessarily the Killing form) to be invariant with respect to $\mathfrak{g}_{0}$ and, moreover, to be non-degenerate such that its inverse $\kappa_{i j}$ exists. The latter is a crucial ingredient in the definition

$$
\left[S_{1}^{a}, S_{2 b}\right]=-\left(R^{i}\right)^{a}{ }_{b} \kappa_{i j} K^{j}
$$

The structure constants which appear in this relation are uniquely determined by the requirement that the metric (2.3) is invariant, i.e. $\langle[X, Y], Z\rangle=\langle X,[Y, Z]\rangle$. The supersymmetry and nondegeneracy of the metric on the full Lie superalgebra $\mathfrak{g}$ follow immediately from the definition.

The commutation relations above preserve the fermion number $\#\left(S_{1}^{a}\right)-\#\left(S_{2 a}\right)$. Hence $\mathfrak{g}$ and also its universal enveloping superalgebra $\mathcal{U}(\mathfrak{g})$ have a natural $\mathbb{Z}$-grading (localized in three degrees) which is consistent with the intrinsic $\mathbb{Z}_{2}$-grading [50]. It is this property which distinguishes type I Lie superalgebras among all Lie superalgebras. Let us also emphasize that the Cartan subalgebra of $\mathfrak{g}$ will always be identified with that of $\mathfrak{g}_{0}$ in what follows. This will be important below when we introduce highest weight representations.

Before we end this subsection on the definition of type I superalgebras, let us reflect a bit on how restrictive their structure is. In fact, in building a Lie superalgebra one cannot just come up with any bosonic subalgebra $\mathfrak{g}_{0}$ and hope to extend it by adding fermions transforming in some representation $R$ of $\mathfrak{g}_{0}$. There is an additional constraint, namely the graded Jacobi identity. While the latter is by assumption identically satisfied for $\mathfrak{g}_{0}$ and the mixed bosonic/fermionic commutators do not impose any new conditions, there is a non-trivial restriction arising from the commutator $\left[S_{1}^{a},\left[S_{1}^{b}, S_{2 c}\right]\right]$ and its cyclic permutations. This leads to the requirement

$$
\left(R^{i}\right)_{c}^{b} \kappa_{i j}\left(R^{j}\right)_{d}^{a}+\left(R^{i}\right)_{c}^{a} \kappa_{i j}\left(R^{j}\right)_{d}^{b}=0
$$

An equivalent formulation is to demand that the quadratic Casimir vanishes on the symmetric part of the tensor product $R \otimes R$. Alternatively, the constraint on the choice of $R$ may be rephrased by requiring that the tensor

$$
A^{a b}{ }_{c d}=\left(R^{i}\right)^{a}{ }_{c} \kappa_{i j}\left(R^{j}\right)^{b}{ }_{d}
$$

is antisymmetric in the upper two as well as the lower two indices. Although the property (2.5) (or (2.6) ) looks rather innocent it will be a crucial ingredient in many of the equalities we shall encounter.

\subsection{Representation theory}

In the analysis of supergroup WZNW models there are a variety of representations of the underlying Lie superalgebra which play a role. The aim of this section is to provide a brief summary of the relevant modules of finite dimensional type I Lie superalgebras following Zou's exposition [48. For definiteness, all the definitions and statements that follow below will be formulated for finite dimensional representations. It is understood, though, that our definitions can be extended to infinite dimensional representations (discrete and continuous) as well. Whether this is also true for their properties, however, remains to be investigated. 


\subsubsection{Kac modules and their duals}

Let us denote by $\operatorname{Rep}\left(\mathfrak{g}_{0}\right)$ the set of isomorphism classes of irreducible representations of the bosonic subalgebra $\mathfrak{g}_{0}$. The basic building blocks in the representation theory of Lie superalgebras of type I are Kac modules $\mathcal{K}_{\mu}, \mu \in \operatorname{Rep}\left(\mathfrak{g}_{0}\right)$ [45, 50]. They are induced from irreducible representations $V_{\mu}$ of the bosonic subalgebra $\mathfrak{g}_{0}$. More precisely, the representation is extended by letting one multiplet of fermionic generators $S_{2 a}$ act trivially on the vectors $v \in V_{\mu}$. The remaining states in the Kac module are then created by acting with generators from the second multiplet of fermions, $S_{1}^{a}$. From our verbal description we immediately infer the decomposition of Kac modules with respect to the bosonic subalgebra 5

$$
\left.\mathcal{K}_{\mu}\right|_{\mathfrak{g}_{0}}=V_{\mu} \otimes \mathcal{F}=\bigoplus_{\nu}\left[\mathcal{K}_{\mu}: V_{\nu}\right]_{0} V_{\nu} .
$$

Here and in what follows we assume all $\mathfrak{g}_{0}$-modules to be fully reducible and denote the resulting multiplicities in terms of the square bracket $\left[M_{0}: N_{0}\right]_{0}$ where $M_{0}$ is an arbitrary (fully reducible) $\mathfrak{g}_{0}$-module and $N_{0}$ an irreducible $\mathfrak{g}_{0}$-module. The $\mathfrak{g}_{0}$-module $\mathcal{F}=\bigwedge\left(S_{1}^{a}\right)$ appearing in the previous equation is the exterior (or Grassman) algebra generated by the fermions $S_{1}^{a}$. Its structure as a $\mathfrak{g}_{0^{-}}$ module is determined by projecting tensor powers of the module $R^{*}$ onto their fully anti-symmetric submodules,

$$
\mathcal{F}=V_{0}^{*} \oplus R^{*} \oplus\left[R^{*} \otimes R^{*}\right]_{\text {antisym }} \oplus\left[R^{*} \otimes R^{*} \otimes R^{*}\right]_{\text {antisym }} \oplus \cdots \oplus\left[\left(R^{*}\right)^{\otimes r}\right]_{\text {antisym }} .
$$

The $n$-fold tensor product here corresponds to a state involving $n$ fermionic generators $S_{1}^{a_{i}}, i=$ $1, \ldots, n$. The case of no fermionic generators leads to the one-dimensional trivial representation $V_{0}=V_{0}^{*}$. It is obvious that the series will truncate after the $r$-th tensor product since the fermionic generators $S_{1}^{a}$ anti-commute among themselves. Consequently, the dimension of Kac modules is always given by $\operatorname{dim}\left(\mathcal{K}_{\mu}\right)=2^{r} \operatorname{dim}\left(V_{\mu}\right)$.

In close analogy to the previous definition we may also introduce dual Kac modules $\mathcal{K}_{\mu}^{*}$ by starting with the dual bosonic representation $V_{\mu}^{*}=V_{\mu^{+}}$. Deviating from the above construction we now let the first set of fermionic generators $S_{1}^{a}$ act trivially on the corresponding vectors and use $S_{2 a}$ to create new states. Since the two sets of fermionic generators transform in dual representations the bosonic content is then obviously given by

$$
\left.\mathcal{K}_{\mu}^{*}\right|_{\mathfrak{g}_{0}}=V_{\mu}^{*} \otimes \mathcal{F}^{*}=\left(V_{\mu} \otimes \mathcal{F}\right)^{*}=\bigoplus_{\nu}\left[\mathcal{K}_{\mu}^{*}: V_{\nu}\right]_{0} V_{\nu}
$$

The dimensions of the modules $\mathcal{K}_{\mu}$ and $\mathcal{K}_{\mu}^{*}$ coincide and it may easily be seen that the representations are indeed dual to each other.

Let us conclude the discussion of Kac modules with a short comment about the last term in the fermionic representation $\mathcal{F}$, eq. (2.8). Innocent as it seems, it is important to stress that the highest component $\left[R^{\otimes r}\right]_{\text {antisym }}$ need not be the trivial $\mathfrak{g}_{0}$-module $V_{0}$ again, even though it certainly is one-dimensional. The action of the bosonic subalgebra on this space can be calculated explicitly,

$$
K^{i} \cdot\left(S_{1}^{1} \cdots S_{1}^{r}\right)=-\operatorname{tr}\left(R^{i}\right) S_{1}^{1} \cdots S_{1}^{r} .
$$

In case $\mathfrak{g}_{0}$ is semisimple, it admits a unique one-dimensional representation, namely the trivial $\mathfrak{g}_{0}$-module $V_{0}$. Hence, we conclude that $\operatorname{tr}\left(R^{i}\right)=0$ for Lie superalgebras with a semisimple bosonic subalgebra.

\footnotetext{
${ }^{5}$ In the following we shall refer to the right hand side of this equation and other restrictions of $\mathfrak{g}$-modules to the bosonic subalgebra $\mathfrak{g}_{0}$ as the "bosonic content". Hence, this phrase is not related to the $\mathbb{Z}_{2}$-grading of the representation space.
} 


\subsubsection{Simple modules and their blocks}

Kac modules provide an important intermediate step to constructing irreducible representations. Finding their exact relation with irreducibles, however, requires good control over the structure of Kac modules. For generic labels $\mu$, the (dual) Kac modules turn out to be irreducible. Thereby, they give rise to what is known as typical irreducible representations $\mathcal{L}_{\mu}=\mathcal{K}_{\mu}$. But there exist special values of $\mu$ for which the associated Kac module contains a proper invariant subspace. The socalled atypical irreducible representations $\mathcal{L}_{\mu}$ are obtained from such $\mathcal{K}_{\mu}$ by factoring out the unique maximal invariant submodule [50]. In contrast to the typical case, it is not straightforward to give a general formula for the dimension or the bosonic content of atypical irreducible representations, see however [48, 51]. As will be explained below the representations $\mathcal{L}_{0}$ as well as $\mathcal{L}_{R}$ and $\mathcal{L}_{R}^{*}=\mathcal{L}_{R^{*}}$ are always atypical.

We shall assume that all irreducible representations of our type I superalgebra $\mathfrak{g}$ emerge as (possibly trivial) quotients of Kac modules (cf. [50]). In other words, the set $\operatorname{Rep}(\mathfrak{g})$ of isomorphism classes (or labels) of irreducible $\mathfrak{g}$-modules agrees with the one of its bosonic subalgebra, i.e. $\operatorname{Rep}(\mathfrak{g})=\operatorname{Rep}\left(\mathfrak{g}_{0}\right)$. According to our previous remarks, it splits into two disjoint sets, $\operatorname{Rep}(\mathfrak{g})=\operatorname{Typ}(\mathfrak{g}) \cup \operatorname{Atyp}(\mathfrak{g})$, containing typical and atypical labels, respectively.

Simple modules of a Lie superalgebra can be grouped into so-called blocks. By definition, blocks are the parts of the finest partition of $\operatorname{Rep}(\mathfrak{g})$ such that two simple modules belong to the same part as soon as they have a non-split extension (see, e.g., [52]). An intuitive way of understanding this definition is to view the simple modules as vertices in a graph. There exists an edge between two vertices if and only if the corresponding simple modules admit a non-split extension. In this picture, the blocks correspond to connected components of the full graph. The property "being connected" defines an equivalence relation $\sim$ on $\operatorname{Rep}(\mathfrak{g})$. We will use the notation $\Gamma(\mathfrak{g})=\operatorname{Rep}(\mathfrak{g}) / \sim$ for the set of all blocks and $[\sigma] \in \Gamma(\mathfrak{g})$ for individual blocks. Notice that each typical module forms a block by itself 6 Atypical irreducible representations, on the other hand, form constituents of larger blocks. This implies the decomposition $\Gamma(\mathfrak{g})=\Gamma_{\text {typ }}(\mathfrak{g}) \cup \Gamma_{\text {atyp }}(\mathfrak{g})$ where $\Gamma_{\text {typ }}(\mathfrak{g})=\operatorname{Typ}(\mathfrak{g})$.

It is easy to argue that each Lie superalgebra of type I possesses a (probably infinite) block [0] containing the trivial representation. Atypicality of the one-dimensional trivial representation already follows on dimensional grounds since the dimension of Kac modules is always a multiple of $2^{r}$. Let us continue to show that the representations $\mathcal{L}_{R}$ and $\mathcal{L}_{R}^{*} \cong \mathcal{L}_{R^{*}}$ which are based on the $\mathfrak{g}_{0}$-modules $R$ and $R^{*}$ lie in the same block [0]. It is straightforward to see that $\mathcal{L}_{0}$ is obtained as a quotient from the Kac module $\mathcal{K}_{0}$ where the subscript 0 refers to the trivial $\mathfrak{g}_{0}$-module. In order to prove the atypicality of $\mathcal{L}_{R}$ we consider the states in $\mathcal{K}_{0}$ which are obtained from the ground state by applying precisely one fermionic generator. These states transform in the representation $R$ of $\mathfrak{g}_{0}$. Since the Kac module $\mathcal{K}_{0}$ is atypical and its irreducible quotient is of dimension one, this representation has to decouple, i.e. the fermionic generators $S_{2 a}$ have to annihilate these states. We observe that the representation $R$ can be part of at least two different supermultiplets: it may be used to define a Kac module $\mathcal{K}_{R}$ and it generates a submodule $\mathcal{Q}_{R}$ of $\mathcal{K}_{0}$. In both cases, the highest weight conditions are exactly identical. But obviously the dimensions of $\mathcal{Q}_{R}$ and $\mathcal{K}_{R}$ do not coincide since $\operatorname{dim} \mathcal{Q}_{R}<\operatorname{dim} \mathcal{K}_{0}<\operatorname{dim} \mathcal{K}_{R}$. Hence, $\mathcal{Q}_{R}$ has to be a non-zero quotient of $\mathcal{K}_{R}$, proving the atypicality of the latter. The same reasoning could be repeated with at least one of the $\mathfrak{g}_{0}$-modules which appear in the (dual) Kac modules $\mathcal{K}_{R}$ and $\mathcal{K}_{R^{*}}$ and so on. Thereby we construct a presumably infinite chain of atypical representations $\mathcal{L}_{\mu}$ in the block [0]. The labels that are

\footnotetext{
${ }^{6}$ This statement only holds in this form if we restrict ourselves to finite dimensional representations.
} 
included in this block all appear in the decomposition of the tensor products $R^{\otimes m} \otimes\left(R^{*}\right)^{\otimes n}$ for arbitrary powers $m$ and $n$ (the converse is not true, of course).

\subsubsection{Projective modules}

Lie superalgebras possess a whole zoo of representations which cannot be decomposed into a direct sum of irreducibles. We shall see some important examples momentarily. Let us recall before that any $\mathfrak{g}$-module $M$ possesses a composition series. The latter is determined by a special kind of filtration, in the present case an ascending set of submodules $M_{i}, i=0, \ldots, n$ where $M_{0}=0$ and $M_{n}=M$, such that the quotients $M_{i} / M_{i-1}$ are simple modules. We will denote by $\left[M: \mathcal{L}_{\mu}\right]$ the number of irreducible $\mathfrak{g}$-modules $\mathcal{L}_{\mu}$ in this composition series of $M$.

The most interesting class of indecomposables consists of the so-called projective covers $\mathcal{P}_{\mu}$ of irreducibles $\mathcal{L}_{\mu}$. The module $\mathcal{P}_{\mu}$ is defined to be the unique indecomposable projective module that contains the irreducible representation $\mathcal{L}_{\mu}$ as its head 7 By definition, the head of a representation is the quotient by its maximal proper submodule. For typical labels one has the equivalences $\mathcal{L}_{\mu} \cong \mathcal{K}_{\mu} \cong \mathcal{P}_{\mu}$. For atypical labels, however, irreducible modules, Kac modules and projective covers are all inequivalent. In particular, they possess different dimensions.

All projective modules $\mathcal{P}$ of a type I superalgebra are known to possess a Kac composition series [48, i.e. a filtration in terms of submodules whose quotients are Kac modules 8 We denote by $\left(\mathcal{P}: \mathcal{K}_{\lambda}\right)$ the number of Kac modules $\mathcal{K}_{\lambda}$ in the Kac composition series of $\mathcal{P}$. In order to describe the precise structure of indecomposable projective modules we will rely on the following reciprocity theorem [48, Theorem 2.7] (see also [49])

$$
\left(\mathcal{P}_{\mu}: \mathcal{K}_{\lambda}\right)=\left[\mathcal{K}_{\lambda}: \mathcal{L}_{\mu}\right]
$$

This important equation relates the multiplicities of Kac modules in the Kac composition series of a projective cover to the multiplicity of irreducible representations arising in the composition series of Kac modules. Hence, the structure of projective covers is completely determined by that of Kac modules. The statement is trivial for typical labels but it contains valuable information in the atypical case. Note that a small technical assumption underlying Zou's proof of eq. (2.11) seems to be overcome if one uses the approach of [49].

There is one simple construction that is guaranteed to furnish projective modules and it is exactly this construction through which the latter will enter in our harmonic analysis later on. The idea is to induce representations from irreducible representations $V_{\mu}$ of $\mathfrak{g}_{0}$ by letting both sets of fermionic generators $S_{1}^{a}$ and $S_{2 a}$ act non-trivially, i.e.

$$
\mathcal{B}_{\mu}=\operatorname{Ind}_{\mathfrak{g}_{0}}^{\mathfrak{g}}\left(V_{\mu}\right)
$$

These modules are projective and reducible [48]. Indeed, under reasonable assumptions on $\mathfrak{g}_{0}$ all finite dimensional $\mathfrak{g}_{0}$-modules are projective, and this property is preserved by induction. For later use, let us write down the decomposition of the representations $\mathcal{B}_{\mu}$ into their indecomposable building blocks. We start with the observation that their bosonic content is given by

$$
\left.\mathcal{B}_{\mu}\right|_{\mathfrak{g}_{0}}=V_{\mu} \otimes \mathcal{F} \otimes \mathcal{F}^{*}
$$

\footnotetext{
${ }^{7}$ The attribute "projective" is used here in the sense of category theory and should not be confused with the notion of projective representations that is used when algebraic relations are only respected up to some multipliers (cocycles).

${ }^{8}$ It should be stressed that this property is not true for type II Lie superalgebras. A counter-example is provided by $D(2,1 ; \alpha)$ whose representation category is discussed in [53].
} 
Using a suitable rearrangement of these factors it is obvious that the multiplicities of Kac modules in the Kac composition series of $\mathcal{B}_{\mu}$ are given by $\left(\mathcal{B}_{\mu}: \mathcal{K}_{\nu}\right)=\left[\mathcal{K}_{\mu^{+}}^{*}: V_{\nu}\right]_{0}$. For the actual decomposition into indecomposables we use our knowledge that $\mathcal{B}_{\mu}$ is projective. This implies that it may be written as a direct sum of (typical) irreducible Kac modules and (atypical) projective covers. While nothing remains to be done for typical representations, the correct description of the atypical sector requires combining the corresponding (non-projective) Kac modules into projective covers. In order to achieve this goal we note the equality $\left[\mathcal{K}_{\mu^{+}}^{*}: V_{\nu}\right]_{0}=\left[\mathcal{K}_{\nu}^{*}: V_{\mu^{+}}\right]_{0}$ which holds because both sides correspond to the number of $\mathfrak{g}_{0}$-invariants in the tensor product $V_{\mu} \otimes V_{\nu}^{*} \otimes \mathcal{F}^{*}$. Now we can use the following simple consequence of the duality relation (2.11),

$$
\left[\mathcal{K}_{\mu^{+}}^{*}: V_{\nu}\right]_{0}=\left[\mathcal{K}_{\nu}^{*}: V_{\mu^{+}}\right]_{0}=\sum_{\sigma}\left[\mathcal{K}_{\nu}^{*}: \mathcal{L}_{\sigma}^{*}\right]\left[\mathcal{L}_{\sigma}^{*}: V_{\mu^{+}}\right]_{0}=\sum_{\sigma}\left(\mathcal{P}_{\sigma}: \mathcal{K}_{\nu}\right)\left[\mathcal{L}_{\sigma}^{*}: V_{\mu^{+}}\right]_{0},
$$

to arrive at the final result

$$
\mathcal{B}_{\mu}=\bigoplus_{\nu \in \operatorname{Typ}(\mathfrak{g})}\left[\mathcal{K}_{\mu^{+}}^{*}: V_{\nu}\right]_{0} \mathcal{K}_{\nu} \oplus \bigoplus_{\sigma \in \operatorname{Atyp}(\mathfrak{g})}\left[\mathcal{L}_{\sigma}^{*}: V_{\mu^{+}}\right]_{0} \mathcal{P}_{\sigma}
$$

This formula will be one of the main ingredients in the harmonic analysis to be performed below in section 4.2. It is interesting to note that every indecomposable projective module arises as a subspaces of some $\mathcal{B}_{\mu}$ [48]. This means that the category of representations considered here "contains enough projectives".

Let us elaborate a bit more on the distinguished role that projective modules - direct sums of typical irreducibles and projective covers of atypical irreducibles - play for the representation theory of Lie superalgebras. In fact, in many ways they take over the role of irreducible representations in the theory of ordinary Lie algebras. Most importantly, it can be shown that the tensor product of any module with a projective one is projective again. In other words, projective modules form an ideal in the representation ring. Moreover, the Clebsch-Gordon decomposition for tensor products of projective modules can be determined through a variant of the Racah-Speiser algorithm. Consider for instance two projective $\mathfrak{g}$-modules $A_{1}$ and $A_{2}$. Being projective, they have a Kac composition series and hence their bosonic content is given by

$$
\left.A_{i}\right|_{\mathfrak{g}_{0}}=\sum_{\mu} m_{i \mu} V_{\mu} \otimes \mathcal{F}
$$

For the bosonic content of the tensor product $A_{1} \otimes A_{2}$ this implies

$$
\left.\left(A_{1} \otimes A_{2}\right)\right|_{\mathfrak{g}_{0}}=\sum_{\mu} m_{1 \mu} m_{2 \nu}\left[V_{\mu} \otimes V_{\nu} \otimes \mathcal{F}\right] \otimes \mathcal{F}
$$

The last $\mathcal{F}$ should be interpreted as the fermionic factor that is guaranteed to be present in every projective module, due to the fact that they possess a Kac composition series. All we need to do is to decompose the factor $V_{\mu} \otimes V_{\nu} \otimes \mathcal{F}$ into irreducibles of $\mathfrak{g}_{0}$. This provides us with a list of all Kac modules in $A_{1} \otimes A_{2}$ along with their multiplicities. Typical Kac modules correspond to irreducible representations appearing in the tensor product while atypical Kac modules must be re-combined into projective covers. This final step is performed based on formula (2.11) and it leads to an unambiguous result. Our discussion shows how the Clebsch-Gordon decomposition of the tensor product $A_{1} \otimes A_{2}$ may be played back to the bosonic subalgebra. The decomposition of $V_{\mu} \otimes V_{\nu} \otimes \mathcal{F}$ can be tackled with the usual algorithmic tools from the representation theory of Lie algebras. 


\subsubsection{The quadratic Casimir element}

One of the most important objects in representation theory are the Casimir elements, i.e. elements of the center of the universal enveloping algebra $\mathcal{U}(\mathfrak{g})$. For our concrete choice of generators and invariant form we have a natural quadratic Casimir

$$
C=K^{i} \kappa_{i j} K^{j}-S_{1}^{a} S_{2 a}+S_{2 a} S_{1}^{a} .
$$

It may easily be checked that this operator acts as a scalar on Kac modules $\mathcal{K}_{\mu}$. For a vector $v \in V_{\mu}$ in the defining irreducible bosonic multiplet one finds

$$
C v=\left(C_{B}-\operatorname{tr}\left(R^{i}\right) \kappa_{i j} K^{j}\right) v
$$

where $C_{B}=K^{i} \kappa_{i j} K^{j}$ is the quadratic Casimir of $\mathfrak{g}_{0}$ associated to its non-degenerate metric. Since the second term inside the bracket commutes with $\mathfrak{g}_{0}$ as well, the irreducibility of $V_{\mu}$ implies that $C$ acts as a scalar on the whole multiplet $V_{\mu}$. Using the commutativity of $C$ with $\mathfrak{g}$, this action may be extended to the complete Kac module $\mathcal{K}_{\mu}$. We will denote the corresponding eigenvalue of the Casimir by $C_{\mu}=C\left(\mathcal{K}_{\mu}\right)$. Because irreducible $\mathfrak{g}$-modules are defined as a quotient of Kac modules this immediately implies $C\left(\mathcal{L}_{\mu}\right)=C\left(\mathcal{K}_{\mu}\right)$.

The observation that several representations may have the same Casimir eigenvalues can be seen to generalize. In fact, it just takes a moment of thought to convince oneself that one has $C_{\mu}=C_{\nu}$ (and the same for other Casimirs) whenever the simple modules belong to the same block, $\mu \sim \nu$. It seems plausible that also the converse holds, i.e. that the set of Casimir operators may be used to separate different blocks. If this assertion was true, then choosing $\mu$ and $\nu$ from different blocks, one would be able to find a Casimir (not necessarily quadratic) whose eigenvalues on $\mathcal{L}_{\mu}$ and $\mathcal{L}_{\nu}$ disagree.

The previous comment that Casimir eigenvalues are constant on blocks has interesting implications for indecomposables. By definition, the composition series of an indecomposable contains irreducibles belonging to one and the same block. Therefore, within any indecomposable, no matter how complicated it is, all generalized eigenvalues of the Casimir elements are the same. The additional qualifier "generalized" is necessary because a Casimir element need not be diagonalizable when evaluated in an indecomposable representation. This phenomenon is particularly common for the projective covers of atypicals. We shall see later that - at least for a type I Lie superalgebra - the quadratic Casimir (2.18) cannot be diagonalized in any of the projective covers $\mathcal{P}_{\mu} 9$ Furthermore, there exists at least one series of projective covers, the ones associated to the block $[0] \in \Gamma(\mathfrak{g})$ of the trivial representation, for which the generalized eigenvalues, i.e. the diagonal entries in the Jordan block, vanish identically 10

\section{The supergroup WZNW model and its symmetries}

In this section we will introduce the WZNW model using its Lagrangian formulation. We will employ a Gauss-like decomposition in order to rewrite the Lagrangian in terms of a bosonic WZNW

\footnotetext{
${ }^{9}$ Diagonalizability might be true for other Casimir operators though. For $g l(1 \mid 1)$, for example, the Casimir element $E^{2}$ is diagonalizable in all weight modules. Note however that $E^{2}$ is not related to a non-degenerate invariant form as in eq. (2.18).

${ }^{10}$ Certain type II superalgebras such as e.g. $D(2,1 ; \alpha)$ are known to also possess projective covers with non-vanishing generalized eigenvalues [53].
} 
model, a free fermion theory and an interaction term. We then describe the infinite dimensional current superalgebra of the model and explain how the latter may be reconstructed from the free fermion resolution introduced before. Let us stress that, contrary to the well known free field approaches to bosonic WZNW models [38, 39, 40, 41, 42], our approach keeps the full bosonic symmetry manifest at all times. It reduces the problem of solving the supergroup WZNW model to a solution of the underlying bosonic model.

\subsection{The Lagrangian description}

Given the Lie superalgebra $\mathfrak{g}$ as defined in (2.1)-(2.4), we can combine its generators with elements of a Grassmann algebra in order to obtain a Lie algebra which can be exponentiated. In physicist's manner we shall define the supergroup $G$ to be given by elements

$$
g=e^{\theta} g_{B} e^{\bar{\theta}}
$$

with $\theta=\theta^{a} S_{2 a}$ and $\bar{\theta}=\bar{\theta}_{b} S_{1}^{b}$ (this parametrization has been termed "chiral superspace" in [54]). The coefficients $\theta^{a}$ and $\bar{\theta}_{b}$ are independent Grassmann variables while $g_{B}$ denotes an element of the bosonic subgroup $G_{B} \subset G$ obtained by exponentiating the Lie algebra generators $K^{i}$. The attentive reader may have noticed that the product of two such supergroup elements (3.1) will not again give a supergroup element of the same form. We shall close an eye on such issues. For us, passing through the supergroup is merely an auxiliary step that serves the purpose of constructing a WZNW-like conformal field theory with Lie superalgebra symmetry. Since Lie superalgebras do not suffer from problems with Grassmann variables, the resulting conformal field theory will be well-defined.

The WZNW Lagrangian for maps $g: \Sigma_{2} \rightarrow G$ from a two-dimensional Riemann surface $\Sigma_{2}$ to the supergroup $G$ is fully specified in terms of the invariant metric on $\mathfrak{g}$ and it reads

$$
\mathcal{S}^{\mathrm{WZNW}}[g]=-\frac{i}{4 \pi} \int_{\Sigma_{2}}\left\langle g^{-1} \partial g, g^{-1} \bar{\partial} g\right\rangle d z \wedge d \bar{z}-\frac{i}{24 \pi} \int_{B_{3}}\left\langle g^{-1} d g,\left[g^{-1} d g, g^{-1} d g\right]\right\rangle .
$$

The second term is integrated over an auxiliary three-manifold $B_{3}$ which satisfies $\partial B_{3}=\Sigma_{2}$. Note that the measure $i d z \wedge d \bar{z}$ is real. The topological ambiguity of the second term possibly imposes a quantization condition on the metric $\langle\cdot, \cdot\rangle$ or, more precisely, on its bosonic restriction, in order to render the path integral well-defined 11 Given the parametrization (3.1), the Lagrangian can be simplified considerably by making iterative use of the Polyakov-Wiegmann identity

$$
\mathcal{S}^{\mathrm{WZNW}}[g h]=\mathcal{S}^{\mathrm{WZNW}}[g]+\mathcal{S}^{\mathrm{WZNW}}[h]-\frac{i}{2 \pi} \int\left\langle g^{-1} \bar{\partial} g, \partial h h^{-1}\right\rangle d z \wedge d \bar{z} .
$$

The WZNW action evaluated on the individual fermionic bits vanishes because the invariant form $(2.3)$ is only supported on grade 0 of the $\mathbb{Z}$-grading. The final result is then

$$
\mathcal{S}^{\mathrm{WZNW}}[g]=\mathcal{S}^{\mathrm{WZNW}}\left[g_{B}, \theta\right]=\mathcal{S}^{\mathrm{WZNW}}\left[g_{B}\right]-\frac{i}{2 \pi} \int\left\langle\bar{\partial} \theta, g_{B} \partial \bar{\theta} g_{B}^{-1}\right\rangle d z \wedge d \bar{z}
$$

\footnotetext{
${ }^{11}$ Note that for WZNW models based on bosonic groups one usually explicitly introduces an integer valued constant, the level, which appears as a prefactor of the Killing form. For supergroups the Killing form might vanish. Hence there is no canonical normalization of the metric. Moreover, we would like to include models whose metric renormalizes non-multiplicatively (see below). Under these circumstances it is not particularly convenient to display the level explicitly and we assume instead that all possible parameters are contained in the metric $\langle\cdot, \cdot\rangle$.
} 
For the correct determination of the mixed bosonic and fermionic term it was again necessary to refer to the grading of $\mathfrak{g}$. The latter implies for instance that the scalar product vanishes if bosonic generators are paired with fermionic ones.

It is now crucial to realize (see also [36, 24]) that we may pass to an equivalent description of the WZNW model above by introducing an additional set of auxiliary fields $p_{a}$ and $\bar{p}^{a}$,

$$
\begin{aligned}
\mathcal{S}\left[g_{B}, p, \theta\right] & =\mathcal{S}_{\text {ren }}^{\mathrm{WZNW}}\left[g_{B}\right]+\mathcal{S}_{\text {free }}[\theta, \bar{\theta}, p, \bar{p}]+\mathcal{S}_{\text {int }}\left[g_{B}, p, \bar{p}\right] \\
& =\mathcal{S}_{\text {ren }}^{\mathrm{WZNW}}\left[g_{B}\right]+\frac{i}{2 \pi} \int\left\{\langle p, \bar{\partial} \theta\rangle-\langle\bar{p}, \partial \bar{\theta}\rangle-\left\langle p, g_{B} \bar{p} g_{B}^{-1}\right\rangle\right\} d z \wedge d \bar{z} .
\end{aligned}
$$

Here, $\theta, \bar{\theta}$ and our new fermionic fields $p=p_{a} S_{1}^{a}$ and $\bar{p}=\bar{p}^{a} S_{2 a}$ all take values in the Lie superalgebra $\mathfrak{g}$. Our conventions may look slightly asymmetric but as we will see later this just resembles the asymmetry in the parametrization (3.1). Up to certain subtleties that are encoded in the subscript "ren" of the first term, it is straightforward to see that we recover the original Lagrangian (3.4) upon integrating out the auxiliary fields $p$ and $\bar{p}$.

Let us comment a bit more on each term in the action (3.5). Most importantly, we need to specify the renormalization of the bosonic WZNW model which results from the change in the path integral measure (cf. [55]). The computation of the relevant Jacobian has two important effects. First of all, it turns out that the construction of the purely bosonic WZNW model entering the action (3.5) employs the following renormalized metric 12

$$
\left\langle K^{i}, K^{j}\right\rangle_{\text {ren }}=\kappa^{i j}-\gamma^{i j} \quad \text { with } \quad \gamma^{i j}=\operatorname{tr}\left(R^{i} R^{j}\right) .
$$

Note that this renormalization is not necessarily multiplicative. For simple Lie superalgebras the renormalized metric is always identical to the original one up to a factor. For non-simple Lie superalgebras, however, this is generically not the case as can be inferred from the example of $g l(1 \mid 1)$.

As a second consequence of the renormalization, the action (3.5) may contain a Fradkin-Tseytlin term, coupling a non-trivial dilaton to the world-sheet curvature $R^{(2)}$,

$$
\mathcal{S}_{\mathrm{FT}}^{\mathrm{WZNW}}\left[g_{b}\right]=\int_{\Sigma_{2}} d^{2} \sigma \sqrt{h} R^{(2)} \phi\left(g_{B}\right) \quad \text { where } \quad \phi\left(g_{B}\right)=-\frac{1}{2} \ln \operatorname{det} R\left(g_{B}\right) .
$$

The same kind of expression has already been encountered in the investigation of the $G L(1 \mid 1)$ WZNW model, cf. [31, 32, 36]. From the discussion at the end of section 2.2.1 it is obvious that $\phi$ vanishes whenever $\mathfrak{g}_{0}$ is a semisimple Lie algebra. Therefore, a non-trivial dilaton is a feature of the series $\operatorname{csp}(2 \mid 2 n), \operatorname{sl}(m \mid n)$ and $g l(m \mid n)$ or, in other words, of most basic Lie superalgebras of type I. The precise reason for the claimed form of renormalization, i.e. the modification of the metric and the appearance of the dilaton, will become clear in the following sections when we discuss the full quantum symmetry of the supergroup WZNW model. At the moment let us just restrict ourselves to the comment that the dilaton is required in order to ensure the supergroup invariance of the path integral measure for the free fermion resolution, i.e. the description of the WZNW model in terms of the Lagrangian (3.5).

\footnotetext{
${ }^{12}$ We assume this metric to be non-degenerate. Otherwise we would deal with what is known as the critical level or, in string terminology, the tensionless limit.
} 
Before we conclude this subsection, let us quickly return to the fermionic terms of the Lagrangian (3.5) which may be rewritten in an even more explicit form using

$$
g_{B} \bar{p} g_{B}^{-1}=g_{B} S_{2 b} \bar{p}^{b} g_{B}^{-1}=S_{2 a} R_{b}^{a}\left(g_{B}\right) \bar{p}^{b} .
$$

The result for the interaction term is

$$
\mathcal{S}_{\text {int }}\left[g_{B}, p, \bar{p}\right]=-\frac{i}{2 \pi} \int p_{a} R_{b}^{a}\left(g_{B}\right) \bar{p}^{b} d z \wedge d \bar{z}
$$

In an operator formulation, the object $R_{b}^{a}\left(g_{B}\right)$ should be interpreted as a vertex operator of the bosonic WZNW model, transforming in the representation $R \otimes R^{*}$. We may consider the interaction term $p_{a} R_{b}^{a}\left(g_{B}\right) \bar{p}^{b}$ as a screening current. Note that the latter is non-chiral by definition, a feature that is not really specific to supergroups but applies equally to bosonic models. Nevertheless, the existing literature on free field constructions did not pay much attention to this point. Actually, the distinction is not really relevant for purely bosonic WZNW models because of their simple factorization into left and right movers. In the present context, however, a complete non-chiral treatment must be enforced in order to capture and understand the special properties of supergroup WZNW models.

\subsection{Covariant formulation of the symmetry}

It is well known that the full WZNW model exhibits a loop group symmetry. More precisely, the Lagrangian (3.2) (and hence also the functional (3.5)) is invariant under multiplication of the field $g(z, \bar{z})$ with holomorphic elements from the left and with antiholomorphic elements from the right. Infinitesimally, each of these transformations generates an infinite dimensional current superalgebra, a central extension $\hat{\mathfrak{g}}$ of the loop superalgebra belonging to $\mathfrak{g}$. For the holomorphic sector the latter is equivalent to the following operator product expansions (OPEs). In the bosonic subsector we find

$$
K^{i}(z) K^{j}(w)=\frac{\kappa^{i j}}{(z-w)^{2}}+\frac{i f^{i j} K^{l}(w)}{z-w} .
$$

The transformation properties of the fermionic currents are

$$
K^{i}(z) S_{1}^{a}(w)=-\frac{\left(R^{i}\right)^{a}{ }_{b} S_{1}^{b}(w)}{z-w} \quad \text { and } \quad K^{i}(z) S_{2 a}(w)=\frac{S_{2 b}(w)\left(R^{i}\right)^{b}{ }_{a}}{z-w} .
$$

Finally we need to specify the OPE of the fermionic currents,

$$
S_{1}^{a}(z) S_{2 b}(w)=\frac{\delta_{b}^{a}}{(z-w)^{2}}-\frac{\left(R^{i}\right)^{a}{ }_{b} \kappa_{i j} K^{j}(w)}{z-w} .
$$

The previous operator product expansions are straightforward extensions of the commutation relations (2.1), (2.2) and (2.4). The central extension is determined by the invariant metric (2.3).

The current superalgebra above defines a chiral vertex algebra via the Sugawara construction [56]. As usual, the corresponding energy momentum tensor is obtained by contracting the currents with the inverse of a distinguished invariant and non-degenerate metric. The appropriate fully renormalized (hence the subscript "full-ren") metric is defined by

$$
\begin{aligned}
\left\langle K^{i}, K^{j}\right\rangle_{\text {full-ren }} & =\left(\Omega^{-1}\right)^{i j}=\kappa^{i j}-\gamma^{i j}-\frac{1}{2} f_{n}^{i m} f_{m}^{j n} \\
\left\langle S_{1}^{a}, S_{2 b}\right\rangle_{\text {full-ren }} & =\left(\Omega^{-1}\right)^{a}{ }_{b}=\delta_{b}^{a}+\left(R^{i} \kappa_{i j} R^{j}\right)^{a}{ }_{b}
\end{aligned}
$$


and it is the result of adding half the Killing form of the Lie superalgebra $\mathfrak{g}$ to the original classical metric (2.3) 13 Note that some of the terms in the fully renormalized metric (3.13) can be identified with the (partially) renormalized metric (3.6) which we introduced while deriving the free fermion Lagrangian. The energy momentum tensor of our theory involves the inverse of the fully renormalized metric,

$$
T=\frac{1}{2}\left[K^{i} \Omega_{i j} K^{j}-S_{1}^{b} \Omega_{b}^{a} S_{2 a}+S_{2 a} \Omega_{b}^{a} S_{1}^{b}\right] .
$$

Both, currents and energy momentum tensor, may similarly be defined for the antiholomorphic sector. The appearance of a renormalized metric in the Sugawara construction is a rather common feature. Supergroup WZNW models are certainly not exceptional in this respect.

In order to complete the discussion of the operator content, we have to introduce vertex operators $\Phi^{(\mathcal{M})}(z, \bar{z})$. The latter carry a representation $\mathcal{M}$ of $\mathfrak{g} \oplus \mathfrak{g}$, the underlying horizontal part of the current superalgebra of our model. If we assume for a moment that $\mathcal{M}=(\mu \nu)$ where $\mu$ and $\nu$ refer to Kac modules of the individual factors in $\mathfrak{g} \oplus \mathfrak{g}$ then primary fields are characterized by the operator products

$$
\begin{aligned}
K^{i}(z) \Phi^{(\mu \nu)}(w, \bar{w})=-\frac{D^{(\mu)}\left(K^{i}\right) \Phi^{(\mu \nu)}(w, \bar{w})}{z-w} & S_{2 a}(z) \Phi^{(\mu \nu)}(w, \bar{w})=0 \\
\bar{K}^{i}(\bar{z}) \Phi^{(\mu \nu)}(w, \bar{w})=\frac{\Phi^{(\mu \nu)}(w, \bar{w}) D^{(\nu)}\left(K^{i}\right)}{\bar{z}-\bar{w}} & \bar{S}_{1}^{a}(\bar{z}) \Phi^{(\mu \nu)}(w, \bar{w})=0 .
\end{aligned}
$$

In addition, there are fields $\left(S_{1}^{a_{1}} \cdots S_{1}^{a_{s}} \bar{S}_{2 b_{1}} \cdots \bar{S}_{2 b_{t}} \Phi^{(\mu \nu)}\right)(z, \bar{z})$ which belong to the same representation of the horizontal subsuperalgebra. The matrices $D^{(\mu)}$ are representation matrices of $\mathfrak{g}_{0}$. As usual we may infer the conformal dimension of the primary fields from their operator product expansion with the energy momentum tensor,

$$
\begin{aligned}
& T(z) \Phi^{(\mu \nu)}(w, \bar{w})=\frac{h^{(\mu \nu)} \Phi^{(\mu \nu)}(w, \bar{w})}{(z-w)^{2}}+\frac{\partial \Phi^{(\mu \nu)}(w, \bar{w})}{z-w} \\
& \bar{T}(\bar{z}) \Phi^{(\mu \nu)}(w, \bar{w})=\frac{\bar{h}^{(\mu \nu)} \Phi^{(\mu \nu)}(w, \bar{w})}{(z-w)^{2}}+\frac{\bar{\partial} \Phi^{(\mu \nu)}(w, \bar{w})}{\bar{z}-\bar{w}} .
\end{aligned}
$$

Using the standard techniques one easily finds that the conformal dimensions are given by (renormalized) Casimir eigenvalues,

$$
h^{(\mu \nu)}=\frac{1}{2} C_{\mu}^{\text {full-ren }} \quad \bar{h}^{(\mu \nu)}=\frac{1}{2} C_{\nu}^{\text {full-ren }} .
$$

The corresponding Casimir is given by $C^{\text {full-ren }}=K^{i} \Omega_{i j} K^{j}+\operatorname{tr}\left(\Omega R^{i}\right) \kappa_{i j} K^{j}$ and should be thought of as a renormalization of eq. (2.19). It is important to stress once more that in our conventions the level is contained implicitly in the metric $\kappa^{i j}$. Thus the conformal dimensions depend on the level. They vanish if the metric of the supergroup is scaled to infinity. In that limit the ground state sector decouples, and it can be analyzed using methods of harmonic analysis. This will be carried out in section 4 .

\footnotetext{
${ }^{13}$ Again, this renormalization does not need to be multiplicative, see for instance $G L(1 \mid 1)$.
} 


\subsection{Free fermion resolution}

Our next aim is to describe the current superalgebra defined above and the associated primary fields in terms of the decoupled system of bosons and fermions that appear in the Lagrangian (3.5). As one of our ingredients we shall employ the bosonic current algebra

$$
K_{B}^{i}(z) K_{B}^{j}(w)=\frac{(\kappa-\gamma)^{i j}}{(z-w)^{2}}+\frac{i f^{i j} K_{B}^{l}(w)}{z-w},
$$

which is defined using the (partially) renormalized metric which has been introduced in (3.6). In addition, we need $r$ free fermionic ghost systems with fields $p_{a}(z)$ and $\theta^{a}(z)$ of spins $h=1$ and $h=0$, respectively. They possess the usual operator products

$$
p_{a}(z) \theta^{b}(w)=\frac{\delta_{a}^{b}}{z-w}
$$

Fermionic fields are assumed to have trivial operator product expansions with the bosonic generators. By construction, the currents $K_{B}^{i}$ and the fields $p_{a}, \theta^{b}$ generate the chiral symmetry of the field theory whose action is

$$
\mathcal{S}_{0}\left[g_{B}, p, \theta\right]=\mathcal{S}_{\mathrm{ren}}^{\mathrm{WZNW}}\left[g_{B}\right]+\mathcal{S}_{\text {free }}[\theta, \bar{\theta}, p, \bar{p}]
$$

Our full WZNW theory may be considered as a deformation of this theory, once we take into account the interaction term between bosons and fermions, see eq. (3.9). The further development of this approach and its consequences will be the subject of section 5 .

But returning first to the decoupled action (3.21), it is easy to see that it defines a conformal field theory with energy momentum tensor

$$
T=\frac{1}{2}\left[K_{B}^{i} \Omega_{i j} K_{B}^{j}+\operatorname{tr}\left(\Omega R^{i}\right) \kappa_{i j} \partial K_{B}^{j}\right]-p_{a} \partial \theta^{a}
$$

Note the existence of the dilaton contributions, i.e. terms linear in derivatives of the currents. In addition to the conformal symmetries, the action (3.21) is also invariant under a $\hat{\mathfrak{g}} \oplus \hat{\mathfrak{g}}$ current superalgebra. The corresponding holomorphic currents are defined by the relations (normal ordering is implied)

$$
\begin{aligned}
K^{i}(z) & =K_{B}^{i}(z)+p_{a}\left(R^{i}\right)^{a}{ }_{b} \theta^{b}(z) \\
S_{1}^{a}(z) & =\partial \theta^{a}(z)+\left(R^{i}\right)_{b}^{a}{ }_{b} \kappa_{i j} \theta^{b} K_{B}^{j}(z)-\frac{1}{2}\left(R^{i}\right)^{a}{ }_{c} \kappa_{i j}\left(R^{j}\right)^{b}{ }_{d} p_{b} \theta^{c} \theta^{d}(z) \\
S_{2 a}(z) & =-p_{a}(z) .
\end{aligned}
$$

It is a straightforward exercise, even though slightly cumbersome and lengthy, to check that this set of generators reproduces the operator product expansions (3.10), (3.11) and (3.12). The only input we need is the Jacobi identity (2.5). The same identity shows that the quantity in (3.23) which is used to contract $p_{b} \theta^{c} \theta^{d}$ is in fact antisymmetric in the lower two indices. Obviously, a similar set of currents may be obtained for the antiholomorphic sector. Given the representation (3.23) for the current superalgebra one may also check the equivalence of the expressions (3.14) and (3.22) for the energy momentum tensors. Algebraically, the calculation rests on the Jacobi identity (2.5) as well as on the equations

$$
\left(\Omega^{-1}\right)^{i j} \kappa_{i j}\left(R^{l}\right)_{b}^{a}=\left(R^{i}\right)_{c}^{a}\left(\Omega^{-1}\right)_{b}^{c}=\left(\Omega^{-1}\right)_{c}^{a}\left(R^{i}\right)_{b}^{c}
$$


The latter arise as invariance constraints for the metric $\langle\cdot, \cdot\rangle_{\text {full-ren }}$ as defined in eq. (3.13).

The current superalgebra defined in (3.23) has a natural action on the vertex operators of the conformal field theory defined by the decoupled Lagrangian $\mathcal{S}_{0}$. Once we include the interaction term, the theory becomes equivalent to the full WZNW model. Hence, we must be able to map the vertex operators of the decoupled theory to the vertex operators of the WZNW theory. The precise relation turns out to be rather involved. Therefore, we postpone a more detailed exposition of this relation to section 5. Instead, we will continue with a semi-classical analysis of the space of vertex operators. This procedure allows us to clearly exhibit the subtleties of the full quantum field theory in a simple and geometric setup.

\section{Semi-classical analysis}

The WZNW model we introduced in the last section admits a semi-classical limit when the invariant metric defined in (2.3) is scaled to infinity. This corresponds to choosing the levels of the underlying bosonic WZNW model large. In this weak curvature regime we expect the conformal dimensions of all primary fields to tend to zero and the higher modes to decouple. We will start with a discussion of the global symmetry of the WZNW model and how it is realized in terms of differential operators on the space of quantum mechanical wave functions. Then we discuss the Laplacian, i.e. the wave operator, on $G$ and determine its (generalized) eigenfunctions and eigenvalues which approximate the vertex operators and their conformal dimensions in the full conformal field theory. It is shown that the spectrum contains non-chiral indecomposable modules on which the Laplacian is not diagonalizable.

\subsection{Symmetry}

One of the inherent properties of supergroup manifolds $G$ is that they admit two actions of $G$ on itself. These so-called left and right regular actions are defined by the maps

$$
L_{h}: \quad g \mapsto h g \quad \text { and } \quad R_{h}: \quad g \mapsto g h^{-1} .
$$

Since the definition of the WZNW Lagrangian (3.2) only involves the invariant metric, both actions are automatically symmetries of our model. In fact, in the present situation they are even promoted to current superalgebra symmetries as we have already seen in the previous section. In this section we will just discuss the point-particle limit (or minisuperspace approximation) where only the zeromodes are taken into account and every dependence on the world-sheet coordinates is ignored. This corresponds to quantum mechanics on the supergroup [57]. Our aim is to find all the eigenfunctions of the Laplace (or wave) operator.

Given the symmetry above we know that the state space of the physical system may be decomposed into representations of $\mathfrak{g} \oplus \mathfrak{g}$. The corresponding symmetry can be realized in terms of differential operators acting on the wave functions which are elements of some algebra of functions $\mathcal{F}(G)$ on the supergroup 14 These functions will naturally depend on a bosonic group element $g_{B}$ and on the fermionic coordinates $\theta^{a}$ and $\bar{\theta}_{a}$. By using a Taylor expansion with respect to the

\footnotetext{
${ }^{14}$ The naive definition of the algebra of function as elements of the Grassmann algebra in the fermions $\theta^{a}$ and $\bar{\theta}_{a}$ with square integrable coefficient functions on $G_{B}$ leads to inconsistencies. A more detailed discussion of these subtle points and the explicit introduction of the correct algebra of function shall be postponed until section 4.3
} 
fermionic variables the basis elements of $\mathcal{F}(G)$ may be represented as a complex valued function depending solely on $g_{B}$ multiplied by a product of Grassmann variables.

The left and right regular action of the supergroup on itself, as given in (4.1), then induces the action

$$
\left(h_{L} \times h_{R}\right) \cdot f: \quad g \mapsto f\left(h_{L}^{-1} g h_{R}\right)
$$

on arbitrary elements $f \in \mathcal{F}(G)$. This in turn translates into the following differential operators,

$$
\begin{aligned}
& K^{i}=K_{B}^{i}-\left(R^{i}\right)^{a}{ }_{b} \theta^{b} \partial_{a} \\
& S_{1}^{a}=R^{a}{ }_{b}\left(g_{B}\right) \bar{\partial}^{b}+\left(R^{i}\right)^{a}{ }_{b} \theta^{b} \kappa_{i j} K_{B}^{j}-\frac{1}{2}\left(R^{i}\right)^{a}{ }_{c} \kappa_{i j}\left(R^{j}\right)^{b}{ }_{d} \theta^{c} \theta^{d} \partial_{b},
\end{aligned}
$$

for the infinitesimal left regular action. In addition to the various structure constants of the Lie superalgebra, these expressions contain derivatives $\partial_{a}=\partial / \partial \theta^{a}$ and $\bar{\partial}^{a}=\partial / \partial \bar{\theta}_{a}$ with respect to the Grassman variables $\theta^{a}$ and $\bar{\theta}_{a}$. We have also introduced the differential operators $K_{B}^{i}$ which implement the regular action of the bosonic subgroup $G_{B}$. They involve derivatives with respect to bosonic coordinates only, but the precise form depends on the particular choice of coordinates on $G_{B}$. Similar expressions can be found for the infinitesimal generators of the right action,

$$
\begin{aligned}
& \bar{K}^{i}=\bar{K}_{B}^{i}+\bar{\theta}_{a}\left(R^{i}\right)^{a}{ }_{b} \bar{\partial}^{b} \quad \bar{S}_{1}^{a}=\bar{\partial}^{a} \\
& \bar{S}_{2 a}=-R_{a}^{b}\left(g_{B}\right) \partial_{b}-\bar{\theta}_{b}\left(R^{i}\right)^{b}{ }_{a} \kappa_{i j} \bar{K}_{B}^{j}-\frac{1}{2}\left(R^{i}\right)^{c}{ }_{a} \kappa_{i j}\left(R^{j}\right)^{d}{ }_{b} \bar{\theta}_{c} \bar{\theta}_{d} \bar{\partial}^{b} .
\end{aligned}
$$

One can check explicitly that these two sets of differential operators form two (anti)commuting copies of the Lie superalgebra $\mathfrak{g}$. Again, these calculations rely heavily on the Jacobi identity (2.5).

The expressions for the differential operators exhibit some peculiar properties that we would like to expand on. Note that, apart from purely bosonic pieces, the generators (4.3) of the left regular action would only involve the Grassmann coordinates $\theta_{a}$ and the corresponding derivatives - but no bared fermions - if it were not for the very first term in the definition of $S_{1}^{a}$. Indeed, this term does contain derivatives with respect to the fermionic coordinates $\bar{\theta}_{a}$. Obviously, the situation is reversed for the right regular action. It is also worth stressing that the coefficients in the first terms of both $S_{1}^{a}$ and $\bar{S}_{2 a}$ are non-trivial functions on the bosonic group. Again this is in sharp contrast to all the other terms whose coefficients are independent of the bosonic coordinates (though functions of the Grassmann variables, of course). It has been emphasized in [24 that the occurrence of the matrix $R\left(g_{B}\right)$ can spoil the normalizability properties of the functions the symmetry transformations are acting on. This always happens if the target space is non-compact since $R$ is a finite dimensional representation and hence non-unitary in that case. Consequently, the product of an $L^{2}$-function from $\mathcal{F}\left(G_{B}\right)$ with $R\left(g_{B}\right)$ will not be an $L^{2}$-function anymore.

In view of these issues with $S_{1}^{a}$ and $\bar{S}_{2 a}$ it is tempting to simply drop the troublesome terms. Even though that might seem a rather arbitrary modification at first, it turns out that the corresponding truncated differential operators $\mathbb{K}^{i}=K^{i}, \mathbb{S}_{2 a}=S_{2 a}$,

$$
\mathbb{S}_{1}^{a}=\left(R^{i}\right)_{b}^{a} \theta^{b} \kappa_{i j} K_{B}^{j}-\frac{1}{2}\left(R^{i}\right)_{c}^{a} \kappa_{i j}\left(R^{j}\right)^{b}{ }_{d} \theta^{c} \theta^{d} \partial_{b}
$$

and their bared analogues also satisfy the commutation relations of $\mathfrak{g} \oplus \mathfrak{g}$ ! For the special case of $P S U(1,1 \mid 2)$, it was explained in [24] that this is much more than a mere curiosity. Indeed, we 
conclude that the truncated operators $\mathbb{K}^{i}, \mathbb{S}_{1}^{a}$ and $\mathbb{S}_{2 a}$ model the action of zero-modes of our currents (3.23) on ground states in the decoupled free fermion theory, i.e. before the coupling of bosonic and fermionic fields is taken into account. Note that the zero-mode of $p(z)$ is a field theoretic incarnation of the derivative $\partial$ since $p(z)$ is the canonically conjugate momentum belonging to $\theta(z)$. We shall now proceed to argue that the original differential operators (4.3) and (4.4) encode a much more intricate structure, namely the action of the zero-modes on primaries in the full interacting WZNW model.

\subsection{Harmonic analysis}

The algebra of functions $\mathcal{F}(G)$ furnishes a representation of $\mathfrak{g} \oplus \mathfrak{g}$ via the differential operators (4.3) and (4.4). Our aim is to write $\mathcal{F}(G)$ as a direct sum of indecomposable building blocks of the type discussed in section 2.2. The final result can be found in eq. (4.7) below. But since the outcome is rather complicated and somewhat hard to digest we would like to start the harmonic analysis by discussing the left and the right action of $\mathfrak{g}$ separately. We claim that the space of functions decomposes under these actions according td 15

$$
\left.\mathcal{F}(G)\right|_{\mathfrak{g}(\text { left })}=\left.\mathcal{F}(G)\right|_{\mathfrak{g}(\text { right })}=\bigoplus_{\mu \in \operatorname{Typ}(G)} \operatorname{dim}\left(\mathcal{K}_{\mu}\right) \mathcal{K}_{\mu} \oplus \bigoplus_{\mu \in \operatorname{Atyp}(G)} \operatorname{dim}\left(\mathcal{L}_{\mu}\right) \mathcal{P}_{\mu}
$$

The symbols $\operatorname{Typ}(G)$ and $\operatorname{Atyp}(G)$ denote the sets of typical and atypical irreducible representations of the supergroup. The distinction between modules of $G$ and modules of $\mathfrak{g}$ is necessary since there might exist representations of the Lie superalgebra which cannot be lifted to $G$. Under rather general conditions (to be recalled below eq. (4.13)) the set $\operatorname{Rep}(G)$ of supergroup representations coincides with $\operatorname{Rep}\left(G_{B}\right) \subset \operatorname{Rep}\left(\mathfrak{g}_{0}\right)$, the set of all unitary irreducible representations of the bosonic subgroup $G_{B}$.

As we see, the decomposition (4.6) clearly distinguishes between the typical and the atypical sector of our space. In the typical sector we sum over irreducible Kac modules $\mathcal{K}_{\mu}=\mathcal{L}_{\mu}$ with a multiplicity space $\mathcal{M}\left(\mathcal{K}_{\mu}\right)$ of dimension $\operatorname{dim} \mathcal{K}_{\mu}$, a prescription which is familiar from the PeterWeyl theory for bosonic groups. In contrast, the atypical sector consists of a sum over all the projective covers $\mathcal{P}_{\mu}$ belonging to atypical irreducibles $\mathcal{L}_{\mu}$ and coming with a multiplicity space $\mathcal{M}\left(\mathcal{P}_{\mu}\right)$ of the smaller dimension $\operatorname{dim} \mathcal{L}_{\mu}<\operatorname{dim} \mathcal{K}_{\mu}$. Note that the algebra of functions forms a projective module and hence possesses a Kac composition series, i.e. a filtration in terms of Kac modules. This immediately permits us to spell out the character of the $\mathfrak{g} \oplus \mathfrak{g}$-module $\mathcal{F}(G)$ and it will lead to a concrete proposal for the modular invariant partition function of the WZNW model in section 5 ,

Naturally, our formula (4.6) is the same for the left and the right action. This symmetry between left and right regular transformations must certainly be maintained when we extend our analysis to the combined left and right action of $\mathfrak{g} \oplus \mathfrak{g}$ on $\mathcal{F}(G)$. In the typical sector the multiplicity spaces of the Kac modules have precisely the dimension that is needed to promote them to Kac modules themselves, a prescription that is perfectly consistent with the symmetry between left and right action. On the other hand, the same symmetry requirement excludes that the individual multiplicity spaces in the atypical sector are simply promoted to irreducible representations of $\mathfrak{g}$. Consequently, the left action must induce maps between various multiplicity spaces for the right action and vice versa. In this way, the atypical sector then consists of non-chiral indecomposables

\footnotetext{
${ }^{15} \mathrm{~A}$ similar expression already appeared in 58 in a more general context.
} 
$\mathcal{I}_{[\sigma]}$ which entangle a (possibly infinite) number of left and right projective covers whose labels belong to the same block $[\sigma]$. The final expression for the representation content of the algebra of functions on $G$ is thus of the form

$$
\left.\mathcal{F}(G)\right|_{\mathfrak{g} \oplus \mathfrak{g}}=\bigoplus_{\mu \in \operatorname{Typ}(G)} \mathcal{L}_{\mu} \otimes \mathcal{L}_{\mu}^{*} \oplus \bigoplus_{[\sigma] \in \Gamma_{\text {atyp }}(G)} \mathcal{I}_{[\sigma]}
$$

The systematic study of the non-chiral representations $\mathcal{I}_{[\sigma]}$ will be left for future work. Note that similar and, in the specific cases of $G L(1 \mid 1)$ and $S U(2 \mid 1)$, more explicit expressions have been obtained in [36, 24, 37]. We also wish to emphasize that the socle of (4.7), i.e. its maximal semisimple subspace, corresponds to a direct sum over all pairs of irreducible representations and their duals. It would be interesting to compare our findings with the more abstract results in [59] where the space of functions on $G=G L(m \mid n)$ is treated in the framework of Hopf superalgebras.

Having stated the main results of this subsection we would like to sketch their derivation. For the proof of eq. (4.6), it is advantageous to enlarge the symmetry from $\mathfrak{g}$ to an action $\mathfrak{g} \oplus \mathfrak{g}_{0}$, i.e. to retain the bosonic generators of the right regular transformations if we analyze the left action. With respect to the combined action one finds

$$
\left.\mathcal{F}(G)\right|_{\mathfrak{g} \oplus \mathfrak{g}_{0}}=\left.\bigoplus_{\mu \in \operatorname{Rep}\left(G_{B}\right)} \mathcal{B}_{\mu} \otimes V_{\mu}^{*} \quad \mathcal{F}(G)\right|_{\mathfrak{g}_{0} \oplus \mathfrak{g}}=\bigoplus_{\mu \in \operatorname{Rep}\left(G_{B}\right)} V_{\mu} \otimes \mathcal{B}_{\mu}^{*}
$$

In fact, from the Peter-Weyl theorem for compact semisimple Lie groups (or suitable generalizations thereof) we deduce that the functions

$$
\operatorname{det} R\left(g_{B}^{-1}\right)\left[D^{(\mu)}\left(g_{B}\right)\right]_{\beta}^{\alpha} \theta^{1} \cdots \theta^{r} \bar{\theta}_{1} \cdots \bar{\theta}_{r}
$$

involving matrix elements of the representation $D^{(\mu)}$ are part of the spectrum for all unitary irreducible representations $\mu$ of $G_{B}$. The matrix elements of $D^{(\mu)}$ transform in the representation $V_{\mu} \otimes V_{\mu}^{*}$ with respect to $\mathfrak{g}_{0} \oplus \mathfrak{g}_{0}$. Since the product of the remaining factors multiplying $D^{(\mu)}$ is invariant under purely bosonic transformations, we conclude that the set of functions (4.9) transforms in $V_{\mu} \otimes V_{\mu}^{*}$ as well.

All that remains to be done is to augment the action on the left from the bosonic subalgebra $\mathfrak{g}_{0}$ to the entire Lie superalgebra $\mathfrak{g}$. The supersymmetric multiplets we generate from the functions (4.9) by repeated action with all the fermionic generators $S_{1}^{a}$ and $S_{2 a}$ are isomorphic to the representation $\mathcal{B}_{\mu}$ of $\mathfrak{g}$. Similar remarks apply if we consider the action of $\mathfrak{g}_{0} \oplus \mathfrak{g}$. Thereby we have established the decompositions (4.8). In order to proceed from eqs. (4.8) to the decomposition formulas (4.6) the representations $\mathcal{B}_{\mu}$ must be decomposed into their indecomposable building blocks. This is achieved with the help of eq. (2.15) and results in eq. (4.6) after a simple re-summation. Our derivation has actually furnished a slightly stronger result since it determines how the multiplicity spaces decompose with respect to the action of the bosonic subalgebra $\mathfrak{g}_{0}$.

\subsection{Spectrum and generalized eigenfunctions}

Given the decomposition of the algebra of functions into representations of $\mathfrak{g} \oplus \mathfrak{g}$ we can now address our original problem of finding the semi-classical expressions of both the conformal dimensions and the primary fields. In the semi-classical limit, conformal dimensions are given by (half) the eigenvalues of the Casimir operator acting on $\mathcal{F}(G)$. Since we are dealing with a space of functions 
we will refer to the latter as "Laplacian" on the supergroup. The eigenvalues can be read off directly from the decomposition (4.7). In the typical sector the Laplacian is diagonalizable and leads to the eigenvalues $C\left(\mathcal{K}_{\mu}\right)$. On the other hand, the Laplacian ceases to be diagonalizable on the non-chiral representations $\mathcal{I}_{[\sigma]}$. Here, the Casimir may just be brought into Jordan normal form.

The previous paragraph provides a complete solution of the eigenvalue problem but it does not yield explicit formulas for the (generalized) eigenfunctions. Since the latter are semi-classical versions of the primary fields in the full CFT (see section 5 below), it seems worthwhile recalling the elegant construction of eigenfunctions that was presented recently in [24]. The Laplace operator on our supergroup $G$ is given by

$$
\Delta=\frac{1}{2} C=\Delta_{B}-\frac{1}{2} \operatorname{tr}\left(R^{i}\right) \kappa_{i j} K_{B}^{j}-\partial_{a} R_{b}^{a}\left(g_{B}\right) \bar{\partial}^{b} .
$$

Observe that only the last term contains fermionic derivatives, with coefficents which depend on bosonic coordinates. Let us also emphasize that the purely bosonic piece of $\Delta$ differs from the Laplacian on the bosonic subgroup by the second term. This deviation is related to the presence of the non-trivial dilaton contribution (3.7). Since the complete Laplacian is non-diagonalizable it was proposed in [24] to perform the harmonic analysis in two steps. First an auxiliary problem is solved which is based on the purely bosonic Laplacian

$$
\Delta_{0}=\Delta_{B}-\frac{1}{2} \operatorname{tr}\left(R^{i}\right) \kappa_{i j} K_{B}^{j}
$$

This auxiliary Laplacian agrees with the Casimir operator obtained from the reduced differential operators $\mathbb{K}$ and $\mathbb{S}$ and, as we shall see, it is completely diagonalizable on the following auxiliary spact 16

$$
\mathbb{F}(G)=\mathcal{F}\left(G_{B}\right) \otimes \bigwedge\left(\theta^{a}, \bar{\theta}_{b}\right)
$$

Here, the factor $\mathcal{F}\left(G_{B}\right)$ denotes the algebra of square (or $\delta$-function) normalizable functions on the bosonic subgroup and $\bigwedge\left(\theta^{a}, \bar{\theta}_{b}\right)$ is the Grassmann (or exterior) algebra generated by the fermionic coordinates. In the second step, the eigenfunctions of $\Delta_{0}$ are mapped to generalized eigenfunctions of $\Delta$ using a linear map $\Xi: \mathbb{F}(G) \rightarrow \mathcal{F}(G)$. The latter adds "subleading" fermionic contributions in a formal but well-defined way and thereby turns an eigenfunction of $\Delta_{0}$ into a generalized eigenfunction of $\Delta$. Our prescription involves explicit multiplications with the matrix elements of $R\left(g_{B}\right)$ which, e.g. for non-compact groups $G_{B}$, are not necessarily part of the unitary spectrum. Hence, the eigenfunctions of $\Delta$ need not be normalizable in the original sense, i.e. when regarded as Grassmann valued functions on the bosonic subgroup. This is the main reason why we need to distinguish between the spaces $\mathbb{F}(G)$ and $\mathcal{F}(G)=\operatorname{Im}(\Xi)$. Ultimately, the problem may be traced back to the presence of the terms involving $R\left(g_{B}\right)$ in $S_{1}^{a}$ and $\bar{S}_{2 a}$. In fact, as we pointed out before, because of those terms the unreduced differential operators may cease to act within $\mathbb{F}(G)$.

In order to gain some intuition into the structure of the function space (4.12) as a representation of the symmetry algebra $\mathfrak{g} \oplus \mathfrak{g}$, it is helpful to restrict the action to the bosonic subalgebra $\mathfrak{g}_{0} \oplus \mathfrak{g}_{0}$ first. Since the differential operators $\mathbb{K}^{i}$ and $\overline{\mathbb{K}}^{i}$ factorize in an action on the function algebra $\mathcal{F}\left(G_{B}\right)$ and on the Grassmann algebra $\bigwedge\left(\theta^{a}, \bar{\theta}_{b}\right)$, we can decompose both factors separately. If the bosonic

\footnotetext{
${ }^{16}$ The auxiliary space $\mathbb{F}(G)$ should be thought of as the semi-classical truncation of the state space for the decoupled theory $\mathcal{S}_{0}$, see eq. (3.21). On the other hand $\mathcal{F}(G)$ corresponds to the semi-classical truncation of the full state space of the WZNW model.
} 
subgroup is compact, semisimple and simply-connected we may employ the Peter-Weyl theorem in order to obtain

$$
\left.\mathcal{F}\left(G_{B}\right)\right|_{\mathfrak{g}_{0} \oplus \mathfrak{g}_{0}}=\bigoplus_{\mu \in \operatorname{Rep}\left(G_{B}\right)} V_{\mu} \otimes V_{\mu}^{*}
$$

where $\operatorname{Rep}\left(G_{B}\right) \subset \operatorname{Rep}\left(\mathfrak{g}_{0}\right)$ is the set of all unitary irreducible representations of $G_{B}$. In more general situations this formula will need a slight refinement concerning the content of $\operatorname{Rep}\left(G_{B}\right)$, although the structure will still be very similar. With regard to the fermions, the left action just affects the set $\theta^{a}$, while the right action operates on the set $\bar{\theta}_{a}$. Given the known transformation behavior of a single fermion we thus find

$$
\left.\bigwedge\left(\theta^{a}, \bar{\theta}_{b}\right)\right|_{\mathfrak{g}_{0} \oplus \mathfrak{g}_{0}}=\mathcal{F} \otimes \mathcal{F}^{*}
$$

Combining these simple facts and defining $\operatorname{Rep}(G)=\operatorname{Rep}\left(G_{B}\right)$ we conclude

$$
\left.\mathbb{F}(G)\right|_{\mathfrak{g}_{0} \oplus \mathfrak{g}_{0}}=\bigoplus_{\mu \in \operatorname{Rep}(G)}\left[V_{\mu} \otimes \mathcal{F}\right] \otimes\left[V_{\mu} \otimes \mathcal{F}\right]^{*}
$$

Before we proceed to the supersymmetric extension, we would like to discuss the general form of elements in the individual subspaces of (4.15). The space of functions is spanned by

$$
f_{b_{1} \cdots b_{t}, \beta}^{(\mu) a_{1} \cdots a_{s}, \alpha}(g)=\left[D^{(\mu)}\left(g_{B}\right)\right]_{\beta}^{\alpha} \theta^{a_{1}} \cdots \theta^{a_{s}} \bar{\theta}_{b_{1}} \cdots \bar{\theta}_{b_{t}}
$$

where $D^{(\mu)}$ denotes the representation of the bosonic subgroup $G_{B}$ on the module $V_{\mu}$.

Our most important task is to determine how the bosonic representations that occur in the decomposition (4.15) combine into multiplets of the full symmetry $\mathfrak{g} \oplus \mathfrak{g}$. As a first hint on what the answer will be, we observe that the representation content in eq. (4.15) agrees with the bosonic content of Kac modules. And indeed, under the action of fermionic generators, the various bosonic modules are easily seen to combine into our modules $\mathcal{K}_{\mu}$. To see this we note that the purely bosonic functions $\left[D^{(\mu)}\left(g_{B}\right)\right]_{\beta}^{\alpha}$ are annihilated by $\mathbb{S}_{2 a}$ and $\overline{\mathbb{S}}_{1}^{a}$ simultaneously and therefore they span the subspace $V_{\mu} \otimes V_{\mu}^{*}$ from which we induce the Kac module $\mathcal{K}_{\mu} \otimes \mathcal{K}_{\mu}^{*}$. Consequently, we obtain the decomposition

$$
\left.\mathbb{F}(G)\right|_{\mathfrak{g} \oplus \mathfrak{g}}=\bigoplus_{\mu \in \operatorname{Rep}(G)} \mathcal{K}_{\mu} \otimes \mathcal{K}_{\mu}^{*}
$$

Note that the sum runs over both typical and atypical representations, i.e. the space of functions is not fully reducible. The Laplacian $\Delta_{0}$ is completely diagonalizable on this space and its eigenvalues are given by eq. (2.19).

Let us now return to the analysis of the space $\mathcal{F}(G)$. We recall that a function $\Phi_{\lambda} \in \mathcal{F}(G)$ is a generalized eigenfunction to the eigenvalue $\lambda$ if there exists a number $n \in \mathbb{N}$ such that

$$
(\Delta-\lambda)^{n} \Phi_{\lambda}=0
$$

Following [24], let us introduce operators $A_{n}(\lambda)$ which are defined through the relation

$$
A_{\lambda}^{(n)}=(\Delta-\lambda)^{n}-\left(\Delta_{0}-\lambda\right)^{n}
$$

In the sequel it will become crucial that each single term of $A_{\lambda}^{(n)}$ contains at least one fermionic derivative. After these preparations we consider a function $f_{\lambda} \in \mathbb{F}(G)$ which is an eigenfunction 
of $\Delta_{0}$, i.e. which satisfies $\Delta_{0} f_{\lambda}=\lambda f_{\lambda}$. We then associate a family of new functions $\Xi_{\lambda}^{(n)} f_{\lambda}$ to $f_{\lambda}$ through

$$
\Xi_{\lambda}^{(n)} f_{\lambda}=\sum_{s=0}^{\infty}\left[-\left(\Delta_{0}-\lambda\right)^{-n} A_{\lambda}^{(n)}\right]^{s} f_{\lambda} \equiv \sum_{s=0}^{r}\left(Q_{\lambda}^{(n)}\right)^{s} f_{\lambda} .
$$

Obviously, the sum truncates after a finite number of terms due to the fermionic derivatives which occur in all the operators $A_{\lambda}^{(n)}$. A formal calculation shows furthermore that the function $\Xi_{\lambda}^{(n)} f_{\lambda}$ is a solution of eq. (4.18). Using the definition (4.20) on each of the eigenspaces $\operatorname{Ker}\left(\Delta_{0}-\lambda\right)$ we obtain a family of maps $\Xi^{(n)}$ which formally exist on the complete function space $\mathbb{F}(G)$.

The only problem with the maps $\Xi^{(n)}$ is that they might be singular on a certain subspace of $\mathbb{F}(G)$. In fact, a close inspection of our expression (4.20) shows that it requires to invert $\left(\Delta_{0}-\lambda\right)$ which may not be possible. If this happens, it signals the existence of functions in $\mathcal{F}(G)$ which are not annihilated by $(\Delta-\lambda)^{n}$ for any $\lambda$, and therefore implies that some Jordan blocks of the Laplacian must have a rank higher than $n$. It may be shown by explicit calculation that the family of maps $\Xi^{(n)}$ stabilizes for $n>r$ and that the resulting limit map $\Xi$ is well-defined on the complete space $\mathbb{F}(G)$ [24]. We then define the space $\mathcal{F}(G)=\operatorname{Im}(\Xi)$ as the image of the auxiliary space $\mathbb{F}(G)$ under $\Xi$. This procedure provides an explicit construction of the eigenspaces and Jordan blocks appearing in the decomposition (4.7). It should also be recalled that the map $\Xi$ acts as an intertwiner between the typical subspace of $\mathbb{F}(G)$ with the reduced action of $\mathfrak{g} \oplus \mathfrak{g}$ and the typical subspace of $\mathcal{F}(G)$ with the full action of $\mathfrak{g} \oplus \mathfrak{g}$ 24]. As before, reduced and full action refer to the use of the differential operators $\left(\mathbb{K}^{i}, \mathbb{S}_{1}^{a}, \mathbb{S}_{2 a}, \overline{\mathbb{K}}^{i}, \overline{\mathbb{S}}_{1}^{a}, \overline{\mathbb{S}}_{2 a}\right)$ and $\left(K^{i}, S_{1}^{a}, S_{2 a}, \bar{K}^{i}, \bar{S}_{1}^{a}, \bar{S}_{2 a}\right)$, respectively.

Within the present context we can actually convince ourselves that the quadratic Casimir is not diagonalizable on any of the projective covers $\mathcal{P}_{\mu}$. From the above it is clear that every projective cover appears in the decomposition of the right regular action on the function space $\mathcal{F}(G)$ and that the corresponding subspace $\mathcal{M}\left(\mathcal{P}_{\mu}\right) \otimes \mathcal{P}_{\mu}$ contains functions of the form (4.9). We claim that some of the latter must necessarily be proper generalized eigenfunctions. In fact, all of them are eigenfunctions of $\Delta_{0}$ with eigenvalue $\lambda=C_{\mu} / 2$. But in order for them to be eigenfunctions of $\Delta$, the action of $\Xi^{(1)}$ must be well defined. This would require in particular that we can invert $\Delta_{0}-\lambda$ on

$$
\partial_{a} R_{b}^{a}\left(g_{B}\right) \bar{\partial}^{b} \operatorname{det} R\left(g_{B}^{-1}\right)\left[D^{(\mu)}\left(g_{B}\right)\right]_{\beta}^{\alpha} \theta^{1} \cdots \theta^{r} \bar{\theta}_{1} \cdots \bar{\theta}_{r} .
$$

But this is clearly not the case if the Kac module $\mathcal{K}_{\mu}$ contains singular vectors that are reached from the ground states through application of a single fermionic generator. Hence, we have established our claim for all such labels $\mu$. In case the singular vectors of $\mathcal{K}_{\mu}$ appear only at higher levels, one has to refine the analysis and consider also higher order (in the summation index $s$ ) terms in the definition of $\Xi^{(1)}$.

\subsection{Correlation functions}

By now we have complete control over representation content and eigenfunctions of the Laplacian in the weak curvature limit of the WZNW model. In addition, we can also compute correlation functions in this limit. They are given as integrals over a product of functions on the supergroup. Integration is performed with an appropriate invariant measure, namely the so-called Haar measure $d \mu(g)$ of the supergroup. The easiest way to obtain $d \mu$ is to extract it from the invariant metric,

$$
d s^{2}=d s_{B}^{2}-2 d \bar{\theta}_{a} R_{b}^{a}\left(g_{B}^{-1}\right) d \theta^{b} .
$$


Here, $d s_{B}^{2}$ denotes the standard invariant metric on the bosonic subgroup. The total metric has a "warped" form since the fermionic bit has an explicit functional dependence on the bosonic coordinates $g_{B}$. We can now obtain the desired measure as the superdeterminant of the metric,

$$
d \mu(g)=d \mu_{B}\left(g_{B}\right) \operatorname{det}\left(R\left(g_{B}\right)\right) d \theta^{1} \cdots d \theta^{r} d \bar{\theta}_{1} \cdots d \bar{\theta}_{r}
$$

where $d \mu_{B}$ denotes an invariant measure on the bosonic subgroup. Once this expression has been written down, we can forget our heuristic derivation and check the invariance explicitly. Note that the existence of the dilaton (3.7) in the WZNW Lagrangian (3.5) is directly related to the presence of the factor $\operatorname{det}\left(R\left(g_{B}\right)\right)$ in the measure.

Suppose now we are given $N$ generalized eigenfunctions of the Laplacian $\Delta$ on the supergroup. According to the previous discussion, the space of eigenfunctions possesses a basis of the form

$$
\begin{gathered}
\phi_{\mu ; \mathbf{b}}^{\mathbf{a}}=\Xi f_{\mu ; \mathbf{b}}^{\mathbf{a}}=\sum_{s=0}^{r} Q_{\lambda}^{s} f_{\mu ; \mathbf{b}}^{\mathbf{a}} \\
\text { where } f_{\mu ; \mathbf{b}}^{\mathbf{a}}=f_{\mu ; b_{1}, \ldots, b_{t}}^{a_{1}, \ldots, a_{s}}=f_{\mu}\left(g_{B}\right) \theta^{a_{1}} \cdots \theta^{a_{s}} \bar{\theta}_{b_{1}} \cdots \theta_{b_{t}} .
\end{gathered}
$$

Here, $f_{\mu}\left(g_{B}\right)$ are eigenfunctions of the bosonic Laplacian $\Delta_{0}$ with eigenvalue $\lambda$ and $\Xi=\Xi^{(r)}, Q_{\lambda}=$ $Q_{\lambda}^{(r)}$ have been defined in eq. (4.20). The $N$-point functions of such semi-classical vertex operators are given by the integrals

$$
\begin{aligned}
\left\langle\phi_{\mu_{1} ; \mathbf{b}_{\mathbf{1}}}^{\mathbf{a}_{1}} \cdots \phi_{\mu_{N} ; \mathbf{b}_{\mathbf{N}}}^{\mathbf{a}_{\mathbf{N}}}\right\rangle & =\int d \mu(g) \phi_{\mu_{1} ; \mathbf{b}_{\mathbf{1}}}^{\mathbf{a}_{\mathbf{1}}} \cdots \phi_{\mu_{N} ; \mathbf{b}_{\mathbf{N}}}^{\mathbf{a}_{\mathbf{N}}} \\
& =\sum_{s_{1}=0}^{r} \cdots \sum_{s_{N}=0}^{r} \int d \mu(g) Q_{\lambda_{1}}^{s_{1}} f_{\mu_{1} ; \mathbf{b}_{\mathbf{1}}}^{\mathbf{a}_{1}} \cdots Q_{\lambda_{N}}^{s_{N}} f_{\mu_{N} ; \mathbf{b}_{\mathbf{N}}}^{\mathbf{a}_{\mathbf{N}}}
\end{aligned}
$$

Most of the $(r+1)^{N}$ terms in this expression vanish due to the properties of Grassmann variables and their integration. In fact the largest number of non-zero terms that can possibly appear is $N \cdot r+1$. This is realized if all eigenfunctions contain terms with the maximal number of fermionic coordinates (along with the lower order terms that are determined by the action of $Q_{\lambda}^{s}$ ). A particularly simple case appears when e.g. the first eigenfunction $\phi_{1}=\phi_{\mu_{1} ; 1,2, \ldots, r}^{1,2, r}$ contains leading terms with $r$ fermions $\theta$ and $\bar{\theta}$ while all others are purely bosonic. In that case, the correlator is simply given by

$$
\left\langle\phi_{\mu_{1} ; 1,2, \ldots, r}^{1,2, \ldots, r} \phi_{\mu_{2}} \cdots \phi_{\mu_{N}}\right\rangle=\int d \mu_{B}\left(g_{B}\right) \operatorname{det}\left(R\left(g_{B}\right)\right) f_{\mu_{1}}\left(g_{B}\right) f_{\mu_{2}}\left(g_{B}\right) \cdots f_{\mu_{N}}\left(g_{B}\right) .
$$

We shall see that very similar results can be established for correlators in the full WZNW on type I supergroups. This is one of the subjects we shall address in the next section.

\section{The quantum WZNW model}

After the thorough discussion of its symmetries and its semi-classical limit it is now only a small step to come up with a complete solution of the full quantum WZNW model. We first show that the free fermion resolution gives rise to a natural class of chiral representations. Subsequently, 
we comment on the representation content of the full non-chiral theory, sketch the calculation of correlation functions and argue that the natural modular invariant partition function can be expressed as a diagonal sum over characters of Kac modules. We conclude with some speculations about non-trivial modular invariants.

\subsection{Chiral representations of the current superalgebra}

In section 3.2 and 3.3 we decribed in some detail the chiral symmetry of WZNW models on supergroups along with their construction in terms of free fermions. Our next aim is to introduce representations $\mathcal{H}_{\mu}$ of $\hat{\mathfrak{g}}$. It is clear that free fermion resolutions provide a natural construction for representations of current superalgebras. What is remarkable, however, is that these representations turn out to be irreducible for generic (typical) choices of $\mu$.

According to the results of section 3.3 every representation of the decoupled system of the bosonic currents $K_{B}^{i}$ and the fermions $p \theta$ defines a module of the current superalgebra via eqs. (3.23). In the bosonic part we shall work with irreducible representations $\mathcal{V}_{\mu}$ of $\hat{\mathfrak{g}}_{0}^{\text {ren }}$. If the group $G_{B}$ is compact there will be a finite number of physical representations (the "integrable" ones), otherwise one may encounter infinitely many of them, including continuous series. We identify the physically relevant representations with a subset $\operatorname{Rep}\left(\hat{\mathfrak{g}}_{0}^{\text {ren }}\right) \subset \operatorname{Rep}\left(\mathfrak{g}_{0}\right)$ within the representation labels for the horizontal subalgebra $\mathfrak{g}_{0}$. This is possible since the ground states of $\mathcal{V}_{\mu}$ form the $\mathfrak{g}_{0}$-module $V_{\mu}$ upon restriction of the $\hat{\mathfrak{g}}_{0}^{\text {ren }}$-action to its horizontal subalgebra $\mathfrak{g}_{0}$. Note that the curvature of the background geometry leads to truncations which imply that $\operatorname{Rep}\left(\hat{\mathfrak{g}}_{0}^{\mathrm{ren}}\right)$ is generally a true subset of $\operatorname{Rep}\left(\mathfrak{g}_{0}\right) 17$ The fermions, on the other hand, admit a unique irreducible representation $\mathcal{V}_{F}$. The latter is generated from the $S L(2, \mathbb{C})$-invariant vacuum $|0\rangle$ by imposing the highest weight conditions $\left(p_{a}\right)_{n}|0\rangle=0$ for $n \geq 0$ and $\theta_{n}^{a}|0\rangle=0$ for $n>018$ The irreducible representations of the product theory therefore take the form

$$
\mathcal{H}_{\mu}=\mathcal{V}_{\mu} \otimes \mathcal{V}_{F}
$$

Given the free fermion realization (3.23), these spaces admit an action of the infinite dimensional current superalgebra $\hat{\mathfrak{g}}$ as defined in (3.10)-(3.12).

The generalized Fock modules $\mathcal{H}_{\mu}$ provide the proper realization of chiral vertex operators as defined around eq. (3.15). It is indeed evident from our construction that the ground states of $\mathcal{H}_{\mu}$ transform in the $\mathfrak{g}$-module $\mathcal{K}_{\mu}$ (recall that the ground states of $\mathcal{V}_{\mu}$ form the $\mathfrak{g}_{0}$-module $V_{\mu}$ ) and that they are annihilated by all positive modes of the currents and by the zero modes of $S_{2 a}(z)$. But there is another and much deeper reason for the relevance of the modules $\mathcal{H}_{\mu}$. Observe that the current superalgebra $\hat{\mathfrak{g}}$ is a true subalgebra of the algebra that is generated from $\hat{\mathfrak{g}}_{0}^{\text {ren }}$ and the fermions. Therefore, one might suspect that the spaces $\mathcal{H}_{\mu}$ are no longer irreducible with respect to the action of $\hat{\mathfrak{g}}$. But for generic choices of $\mu$ this is not the case: The action of $\hat{\mathfrak{g}}$ on $\mathcal{H}_{\mu}$ is typically irreducible! This property is in sharp contrast to what happens for standard bosonic free field constructions [38, [39, 40, 41, 42] and it characterizes the modules $\mathcal{H}_{\mu}$ as the natural infinite dimensional lift of Kac modules for the finite dimensional Lie superalgebra $\mathfrak{g}$. We take this observation as a motivation to refer to the generalized Fock modules $\mathcal{H}_{\mu}$ as Kac modules from now

\footnotetext{
${ }^{17}$ For $\widehat{s u}(2)_{k}$, for instance, the integrable representations are $\lambda=0,1, \ldots, k$ while there is no upper bound for unitary $s u(2)$-modules.

${ }^{18}$ One could include twisted sectors where the moding of the fermions is not integer. But then the global supersymmetry would not be realized in the WZNW model since there were no zero-modes.
} 
on. Let us emphasize, however, that they are constructed in a different manner than those of the finite dimensional Lie superalgebra $\mathfrak{g}$ in section 2.2.1.

Since it is a rather crucial issue for the following, we would like to spend some time to establish irreducibility of the representations $\mathcal{H}_{\mu}$ for generic labels $\mu$. We shall assume for simplicity that the underlying bosonic representation $\mathcal{V}_{\mu}$ is a highest weight module. The highest weight $\mu$ determines two seemingly different (but in fact equivalent) Verma-like modules of $\hat{\mathfrak{g}}$. The first of them will be denoted by $\mathcal{Y}_{\mu}^{\prime}$. It is obtained as a product

$$
\mathcal{Y}_{\mu}^{\prime}=\mathcal{Y}_{\mu}^{(0, \text { ren })} \otimes \mathcal{V}_{F}
$$

of the Verma module $\mathcal{Y}_{\mu}^{(0, \text { ren })}$ of $\hat{\mathfrak{g}}_{0}^{\text {ren }}$ with the free fermion state space $\mathcal{V}_{F}$. We shall consider $\mathcal{Y}_{\mu}^{\prime}$ as a $\hat{\mathfrak{g}}$-module. The $\hat{\mathfrak{g}}$-module $\mathcal{H}_{\mu}$ may be recovered from $\mathcal{Y}_{\mu}^{\prime}$ by dividing out all the bosonic singular vectors from the $\hat{\mathfrak{g}}_{0}^{\text {ren }}$-module $\mathcal{Y}_{\mu}^{(0, \text { ren })}$. But there is a second natural Verma-like module $\mathcal{Y}_{\mu}$ for $\hat{\mathfrak{g}}$ which is constructed directly by requiring that all the positive modes as well as the zeromodes $\left(S_{2 a}\right)_{0}$ annihilate the highest weight, i.e. $\mathcal{Y}_{\mu}$ is defined without any reference to the free fermion construction of $\hat{\mathfrak{g}}$. Since the generators $K_{n}^{i}, S_{1, n}^{a}, S_{2 b, n}$ and $K_{B, n}^{i}, \theta_{n}^{a}, p_{a, n}$ are in one-to-one correspondence with each other, the Verma modules $\mathcal{Y}_{\mu}$ and $\mathcal{Y}_{\mu}^{\prime}$ are naturally isomorphic as vector spaces. The natural isomorphism preserves the grading by conformal dimensions. Hence, the characters of $\mathcal{Y}_{\mu}$ and $\mathcal{Y}_{\mu}^{\prime}$ agree. It is tempting to conjecture that $\mathcal{Y}_{\mu}$ and $\mathcal{Y}_{\mu}^{\prime}$ are in fact equivalent as $\hat{\mathfrak{g}}$-modules.

In order to understand the equality of conformal dimensions we could simply refer to the equivalence of energy momentum tensors which has been proven in section 3.3. But there is also a more pedestrian way of seeing it. In the case of $\hat{\mathfrak{g}}_{0}^{\text {ren }}$, the current algebra involves the renormalized metric $\kappa-\gamma$ while the bosonic subalgebra $\hat{\mathfrak{g}}_{0}$ of $\hat{\mathfrak{g}}$ is defined in terms of the metric $\kappa$. But according to the Sugawara constructions for $\hat{\mathfrak{g}}_{0}^{\text {ren }}$ and $\hat{\mathfrak{g}}$, the respective energy momentum tensor requires an additional quantum renormalization of the metric in both cases. This extra renormalization is different as well and the final result (the "fully renormalized metric") coincides again. The previous statement corresponds to the two different ways of introducing brackets in the following equation,

$$
\left(\kappa^{i j}-\gamma^{i j}\right)-\frac{1}{2} f_{n}^{i m} f_{m}^{j n}=\kappa^{i j}-\left(\gamma^{i j}+\frac{1}{2} f_{n}^{i m} f_{m}^{j n}\right) .
$$

The first term on both sides refers to the "classical" metric and the second term describes the quantum renormalization. In addition, the effect of the fermions in $\hat{\mathfrak{g}}$ has to be traded for the presence of the dilaton in the $\hat{\mathfrak{g}}_{0}^{\text {ren }}$ description.

Let us now focus on the Verma-like modules $\mathcal{Y}_{\mu}$. In general, these modules contain singular vectors, certainly of bosonic type but possibly also fermionic ones. Our goal here is two-fold: First, we would like to argue for a one-to-one correspondence of the bosonic singular vectors with those in $\mathcal{Y}_{\mu}^{(0, \text { ren })}$. Moreover, we would like to show that the existence of fermionic singular vectors is an atypical event, occurring only for a small subset of weights $\mu$.

In principle, the structure of singular vectors in the module $\mathcal{Y}_{\mu}$ can be discussed using a suitable variant of the Kac-Kazhdan determinant [50]. For simplicity we shall follow a more down-to-earth approach here. The existence of a proper submodule $\mathcal{Y}_{\nu}$ in the representation $\mathcal{Y}_{\mu}$ requires that the weight $\nu$ can be reached from $\mu$ by (multiple) application of the root generators of $\hat{\mathfrak{g}}$. We may qualify this further with the help of two gradings, one with respect to the generator $L_{0} 19$ and the

\footnotetext{
${ }^{19}$ The metric or the level(s), respectively, are assumed to be fixed once and for all.
} 
other coming from the Cartan subalgebra of $\mathfrak{g}$ (which is identical to that of $\mathfrak{g}_{0}$ ). The latter implies that the weights $\mu$ and $\nu$ have to be related by $\nu=\mu-m \alpha$ where $\alpha$ is a positive root of $\mathfrak{g}$ and $m \in \mathbb{Z}_{\geq 0}$. If the energy direction is considered separately, one obtains a necessary condition of the form

$$
h_{\mu-m \alpha}=h_{\mu}+n m,
$$

where $h$ denotes the conformal dimension and the root generator belonging to $\alpha$ is assumed to increase the energy by $n$ units.

We will investigate condition (5.3) for bosonic root generators of $\hat{\mathfrak{g}}$ first. The latter are in oneto-one correspondence with those of $\hat{\mathfrak{g}}_{0}^{\text {ren }}$. Since, in addition, the conformal dimensions of highest weight modules $\mathcal{Y}_{\mu}$ and $\mathcal{Y}_{\mu}^{(0, \text { ren })}$ coincide, we conclude that the associated decoupling equations (5.3) possess the same set of bosonic solutions. We consider this a strong hint that singular vectors in the $\hat{\mathfrak{g}}_{0}$-modules $\mathcal{Y}_{\mu}^{(0, \text { ren })} \otimes \mathcal{V}_{F}$ agree with those singular vectors of the $\hat{\mathfrak{g}}$-modules $\mathcal{Y}_{\mu}$ which can be reached by application of bosonic root generators. If we assume this to be true, all bosonic singular vectors are removed when be pass from $\mathcal{Y}_{\mu}$ to $\mathcal{H}_{\mu}$. Therefore, the singular vectors that remain in $\mathcal{H}_{\mu}$ are necessarily fermionic.

Let us now look for the existence of potential fermionic singular vectors. We do not intend to formulate any precise rules for when they appear, but would like to argue that they must be rare compared to their bosonic counterparts. To this end, we recall that the conformal dimension $h$ is a quadratic expression of the form $h_{\mu}=\langle\mu, \mu+2 \rho\rangle$ (the bracket denoting the non-degenerate scalar product that comes with the metric (3.13) $)$. Hence, we can always solve eq. (5.3) for $m$, no matter which bosonic root vector $\alpha$ we insert. This ceases to be true for fermionic root generators. Since they are nilpotent, eq. (5.3) needs to be solved with $m=0,1$, something that rarely ever works out. Therefore, modules with fermionic singular vectors are called atypical. A more systematic study of atypical representations is beyond the scope of this article. But the experience with several examples suggests that the composition series of the representations $\mathcal{H}_{\mu}$ is finite and that they possess the same structure as the modules of the horizontal subsuperalgebra. In fact, we believe that the only possible fermionic singular vectors are those that appear on the level of ground states and images thereof under the action of certain spectral flow automorphisms (see section [5.2).

Given the structure of the Kac modules (5.1) it is straightforward to derive character formulas and their modular properties. Indeed, the characters simply factorize into

$$
\chi_{\mathcal{H}_{\mu}}(q)=\chi_{\mathcal{V}_{\mu}}(q) \chi_{\mathcal{V}_{F}}(q)
$$

The supercharacter of $\mathcal{H}_{\mu}$ has the same product form but with the fermionic factor $\chi_{\mathcal{V}_{F}}$ being replaced by its corresponding supercharacter. Relation (5.4) may also be extended to a statement about non-specialized characters since the fermions $p_{a}$ and $\theta^{a}$ are charged under the bosonic generators $K^{i}$. If $\mathfrak{g}_{0}$ is a simple Lie algebra the characters of the unitary $\hat{\mathfrak{g}}_{0}^{\text {ren }}$-modules $\mathcal{V}_{\mu}$ can be looked up in [60, 61]. They form a finite dimensional unitary representation of the modular group. The character of the fermionic representation $\mathcal{V}_{F}$, on the other hand, is given by

$$
\chi_{\mathcal{V}_{F}}(q)=\left[2 q^{\frac{1}{12}} \prod_{n=1}^{\infty}\left(1+q^{n}\right)^{2}\right]^{r}=\left[\frac{\vartheta_{2}(q)}{\eta(q)}\right]^{r} .
$$

Under the modular transformation $\tau \mapsto-1 / \tau$ the quotient $\vartheta_{2} / \eta$ is simply replaced by $\vartheta_{4} / \eta$. Hence, all the non-trivial information about modular transformations resides in the behaviour of the characters for the bosonic algebra $\hat{\mathfrak{g}}_{0}^{\text {ren }}$. Consequently, the modular properties of Kac modules $\mathcal{H}_{\mu}$ 
are under complete control. Even though Kac modules do not suffice to build the state space of WZNW models on supergroups, the bulk partition function for type I supergroups may be expressed in terms of characters of Kac modules (see below). Therefore, modular invariance of the bulk partition function is guaranteed as long as it involves a summation over the same set of labels as in the corresponding bosonic model. The precise construction will be explained in more detail in section 5.3 .

It remains to work out the characters of atypical irreducible representations. The latter are quotients of reducible Kac modules. According to our experience with concrete models, the composition series of the infinite dimensional Kac modules $\mathcal{H}_{\mu}$ of $\hat{\mathfrak{g}}$ is very closely related to that of Kac modules for the horizontal subsuperalgebra $\mathfrak{g}$. In specific examples it is usually straightforward to invert the linear relations between characters resulting from such a composition series, i.e. to express the characters of atypical irreducible representations through those of Kac modules. A more general approach to this problem using Kazhdan-Lusztig polynomials has been presented in [48, Proposition 5.4] (see also [51, 62]). Recently it has been shown that the solution for the inversion problem could be used to (re)derive the characters of irreducible representations for the affine Lie superalgebras $\widehat{s l}(2 \mid 1)$ and $\widehat{p s l}(2 \mid 2)$ [24, 37]. We expect that this observation extends to more general current superalgebras and that it will be helpful in the study of modular transformations. Representations of affine Lie superalgebras and their behaviour under modular transformations have also been studied in [63, 64, 13].

\subsection{Spectral flow automorphisms}

In the previous subsection we have skipped over one rather important element in the representation theory of current (super)algebras: The spectral flow automorphisms. As we shall recall momentarily, spectral flow automorphisms describe symmetry transformations in the representation theory of current algebras. Furthermore, they seem to be realized as exact symmetries of the WZNW models on supergroups, a property that makes them highly relevant for our discussion of partition functions below.

Throughout the following discussion, we shall denote (spectral flow) automorphisms of the current superalgebra $\hat{\mathfrak{g}}$ by $\omega$. We shall mostly assume that the action of $\omega$ is consistent with the boundary conditions for currents, i.e. that it preserves the integer moding of the currents. In the context of representation theory, any such spectral flow automorphism $\omega$ defines a map on the set of (isomorphism classes of) representations $\rho: \hat{\mathfrak{g}} \rightarrow \operatorname{End}(V)$ via concatenation, $\omega(\rho)=\rho \circ \omega: \hat{\mathfrak{g}} \rightarrow$ $\operatorname{End}(V)$.

In line with our general strategy, we would like to establish that spectral flow automorphisms $\omega$ of the current superalgebra are uniquely determined by their action on the bosonic generators. A spectral flow automorphism $\omega: \hat{\mathfrak{g}}_{0} \rightarrow \hat{\mathfrak{g}}_{0}$ of the bosonic subalgebra $\hat{\mathfrak{g}}_{0}$ is, by definition, a linear map20

$$
\omega\left(K^{i}(z)\right)=\left(W_{0}\right)^{i}{ }_{j}(z) K^{j}(z)+w_{0}^{i} z^{-1}
$$

satisfying certain consistency conditions to be recalled below. The map $W_{0}(z)=z^{\zeta_{0}}$ is defined in terms of an endomorphism $\zeta_{0}: \mathfrak{g}_{0} \rightarrow \mathfrak{g}_{0}$ of the horizontal subalgebra. While the eigenvalues of $\zeta_{0}$ determine how the spectral flow shifts the modes of the currents, the vector $w_{0}^{i}$ affects only the zero-modes. In order to preserve the trivial monodromy under rotations around the origin we

\footnotetext{
${ }^{20}$ We refrain from introducing a different symbol here such as $\omega_{0}$.
} 
will assume that $W_{0}(z)$ is a meromorphic function, i.e. that all the eigenvalues of $\zeta_{0}$ are integer. Inserting the transformation (5.6) into the operator product expansions (3.10) leaves one with the constraints

$$
\left(\zeta_{0}\right)_{j}^{i}=f_{l}^{i k} \kappa_{k j} w_{0}^{l}
$$

and

$$
\left(W_{0}\right)_{k}^{i}(z)\left(W_{0}\right)^{j}{ }_{l}(z) \kappa^{k l}=\kappa^{i j} \quad, \quad f^{i j}{ }_{k}\left(W_{0}\right)^{k}{ }_{l}(z)=\left(W_{0}\right)_{m}^{i}(z)\left(W_{0}\right)^{j}{ }_{n}(z) f_{l}^{m n} .
$$

The first equation (5.7) in fact implies that the only free parameter is the shift vector $w_{0}^{i}$. In the case of a semisimple Lie algebra $\mathfrak{g}_{0}$ (which leads to a non-degenerate Killing form) this argument can also be reversed and hence it allows to express $w_{0}^{i}$ in terms of $\zeta_{0}$.

We would now like to argue that equation (5.7) already implies the consistency of the spectral flow (up to the question whether $\zeta_{0}$ has integer eigenvalues), i.e. the validity of the equations (5.8). Given the concrete form of $W_{0}(z)$, it can indeed be shown that the two relations (5.8) follow from the equations

$$
\left(\zeta_{0}\right)_{k}^{i} \kappa^{k j}+\left(\zeta_{0}\right)^{j}{ }_{l} \kappa^{i l}=0 \quad \quad f^{i j}{ }_{k}\left(\zeta_{0}\right)^{k}{ }_{l}=\left(\zeta_{0}\right)_{k}{ }_{k} f^{k j}{ }_{l}+\left(\zeta_{0}\right)^{j}{ }_{k} f^{i k}{ }_{l} .
$$

These relations are in turn just a consequence of (5.7) using the invariance of $\kappa^{i j}$ and the Jacobi identity for the structure constants. Since the same idea will be used again below let us sketch the proof of our assertion that the eqs. (5.9) imply the eqs. (5.8). First of all, it is easy to see that one can generalize the relations (5.9) to powers of $\zeta_{0}$ using induction. In the first case, this just yields an alternating relative sign, while in the second case it establishes some kind of binomial formula. Writing $W_{0}(z)=\exp \left(\zeta_{0} \ln z\right)$ and expanding in powers of $\ln z$ one can then explicitly verify the equations for $W_{0}(z)$. Any vector $w_{0}^{i}$ which leads to a matrix $\zeta_{0}$ with integer eigenvalues under the identification (5.7) will accordingly be referred to as a spectral flow automorphism of $\hat{\mathfrak{g}}$ from now on.

Given the insights of the previous paragraphs it is now fairly straightforward to extend the spectral flow automorphism $\omega: \hat{\mathfrak{g}}_{0} \rightarrow \hat{\mathfrak{g}}_{0}$ to the full current superalgebra. To this end, we introduce the element

$$
\zeta_{1}=-R^{i} \kappa_{i j} w_{0}^{j}
$$

It is crucial to observe that this matrix satisfies the relation

$$
\left(\zeta_{0}\right)^{i}{ }_{j}\left(R^{j}\right)^{a}{ }_{c}+\left(\zeta_{1}\right)_{b}^{a}\left(R^{i}\right)_{c}^{b}=\left(R^{i}\right)^{a}{ }_{b}\left(\zeta_{1}\right)^{b}{ }_{c}
$$

an analogue of eq. (5.9). Following the discussion in the bosonic sector, we now introduce a function $W_{1}(z)=z^{\zeta_{1}}$. Using the same reasoning as in the previous paragraph, the equation (5.11) implies

$$
\left(R^{i}\right)^{a}{ }_{b}\left(W_{1}\right)^{b}{ }_{c}(z)=\left(W_{0}\right)^{i}{ }_{j}(z)\left(W_{1}\right)^{a}{ }_{b}(z)\left(R^{j}\right)^{b}{ }_{c} \cdot
$$

Now we can define the action of the spectral flow automorphism $\omega$ on the fermionic currents by

$$
\omega\left(S_{1}^{a}(z)\right)=\left(W_{1}\right)_{b}^{a}(z) S_{1}^{b}(z) \quad, \quad \omega\left(S_{2 a}(z)\right)=S_{1 b}(z)\left(\bar{W}_{1}\right)^{b}{ }_{a}(z)
$$

where $\bar{W}_{1}$ denotes the inverse of $W_{1}$. Once more, consistency with the operator product expansions of the supercurrents is straightforward to verify. The only input is the definition (5.10) and the property (5.12). 
We would also like to argue that the spectral flow symmetry is consistent with the free fermion representation (3.23). To be more specific, we shall construct an automorphism on the chiral algebra of the decoupled system generated by the currents $K_{B}^{i}(z)$ and the free fermions $p_{a}(z)$ and $\theta^{a}(z)$ that reduces to the expressions above if we plug the transformed fields into the defining equations (3.23). In this context the most important issue is to understand how the renormalization of the metric $\kappa \rightarrow \kappa-\gamma$ affects the action of the spectral flow. As a consequence of eq. (5.11) we note that

$$
\left(\zeta_{0}\right)_{k}^{i} \gamma^{k j}+\left(\zeta_{0}\right)_{k}^{j} \gamma^{i k}=\operatorname{tr}\left(\left[R^{i} R^{j}, \zeta_{1}\right]\right)=0
$$

where $\gamma^{i j}=\operatorname{tr}\left(R^{i} R^{j}\right)$, as before. Consequently, the data $\zeta_{0}$ which gave rise to a spectral flow automorphism of $\hat{\mathfrak{g}}_{0}$ above, can also be used to define a spectral flow automorphism of the renormalized current algebra, i.e. of the algebra that is generated by $K_{B}^{j}$ with operator products given in subsection (3.3). Only the shift vector $w_{0}^{i}$ of the zero modes needs a small adjustment such that the new spectral flow action reads

$$
\omega\left(K_{B}^{i}(z)\right)=\left(W_{0}\right)^{i}{ }_{j}(z) K_{B}^{j}(z)+w_{B}^{i} z^{-1} \quad \text { where } \quad w_{B}^{i}=w_{0}^{i}+\operatorname{tr}\left(\zeta_{1} R^{i}\right) .
$$

In order to validate that this indeed defines an automorphism we need to check the analogue of the condition (5.7) for the new metric $\kappa-\gamma$. But this constraint is trivially met, using

$$
w_{B}^{i}=(\kappa-\gamma)^{i j} \kappa_{j k} w_{0}^{k}
$$

along with the invariance of both metrics $\kappa$ and $\kappa-\gamma$. Note that $\zeta_{0}$ is not changed and hence it has the same (integer) eigenvalues as before.

In order to obtain an automorphism which is compatible with the free field construction we also need to introduce the transformations

$$
\omega\left(p_{a}(z)\right)=p_{b}(z)\left(\bar{W}_{1}\right)_{a}^{b}(z) \quad, \quad \omega\left(\theta^{a}(z)\right)=\left(W_{1}\right)_{b}^{a}(z) \theta^{b}(z) .
$$

It is then straightforward but lengthy to check that the previous transformations define an automorphism of the algebra generated by $p_{a}, \theta^{a}$ and $K_{B}^{j}$ that descends to the original spectral flow automorphism $\omega$ of our current superalgebra $\hat{\mathfrak{g}}$. During the calculation one has to be aware of normal ordering issues.

In conclusion we have shown that any spectral flow automorphism of the bosonic subalgebra of a current superalgebra (related to a Lie superalgebra of type I) can be extended to the full current superalgebra. Furthermore, this extension was seen to be consistent with our free fermion resolution. Let us remark that even if we start with a spectral flow automorphism $\omega$ preserving periodic boundary conditions for bosonic currents, the lifted spectral flow $\omega$ does not necessarily have the same property on fermionic generators. Only those spectral flow automorphisms $\omega: \hat{\mathfrak{g}} \rightarrow \hat{\mathfrak{g}}$ for which $W_{1}$ is meromorphic as well seem to arise as symmetries of WZNW models on supergroups. Nevertheless, also non-meromorphic spectral flows turn out to be of physical relevance. They can be used to describe the twisted sectors of orbifold theories, see section 5.4 for details.

\subsection{Spectrum and correlation functions}

Obviously, it is of central importance to determine the partition function and higher correlators of WZNW models on supergroups. Here we shall explain how the calculation of these quantities may be reduced to computations in the corresponding bosonic WZNW models. For the torus partition 
function we will provide a full expression in terms of characters of the (renormalized) bosonic current algebra.

All computations in the WZNW model on type I supergroups depart from the decoupled theory (3.21). The interaction between bosons and fermions is treated perturbatively. What makes this approach particularly powerful is the fact that the perturbative expansion turns out to truncate after a finite number of terms. The order at which the truncation occurs, however, depends on the supergroup and the correlator to be computed. As a general rule, the number of terms to consider in the perturbative expansion increases with the number of vertex operators that are inserted.

To begin with, let us describe the unperturbed theory (3.21) with a few concrete formulas. As we proceed it is useful to keep in mind that solving the unperturbed theory is a field theoretic analogue of solving the truncated Laplace operator $\Delta_{0}$. Fields in the decoupled theory form a space $\mathbb{H}$ which is a field theoretic version of the semi-classical space $\mathbb{F}(G)$. The state space $\mathbb{H}$ naturally factorizes into bosonic and fermionic contributions,

$$
\mathbb{H}=\bigoplus_{\mu \in \operatorname{Rep}\left(\hat{\mathfrak{g}}_{0}^{\mathrm{ren}}\right)}\left(\mathcal{V}_{\mu} \otimes \mathcal{V}_{F}\right) \otimes\left(\overline{\mathcal{V}}_{\mu}^{*} \otimes \overline{\mathcal{V}}_{F}\right)
$$

For simplicity we assumed that the bosonic part has a charge conjugate modular invariant partition function 21 The fermionic representation is unique if we restrict ourselves to the Ramond-Ramond sector. In case applications require to include fermionic fields with anti-periodic boundary conditions as well, they can be incorporated easily. According to eq. (5.18), vertex operators of the decoupled theory possess a basis of the form

$$
V_{\mu ; \mathbf{b}}^{\mathbf{a}}(z, \bar{z}) \equiv V_{\mu ; b_{1}, \ldots, b_{t}}^{a_{1}, \ldots, a_{s}}(z, \bar{z})=V_{\mu}(z, \bar{z}) \theta^{a_{1}}(z) \cdots \theta^{a_{s}}(z) \bar{\theta}_{b_{1}}(\bar{z}) \cdots \bar{\theta}_{b_{t}}(\bar{z})
$$

where $V_{\mu}$ are vertex operators in the bosonic WZNW model. We have noted before that the free fermion theory admits a current superalgebra symmetry $\hat{\mathfrak{g}} \oplus \hat{\mathfrak{g}}$. The latter is given explicitly by the formulas in section 3.3. When analyzed with respect to this current superalgebra, the state space $\mathbb{H}$ assumes the form

$$
\mathbb{H}=\bigoplus_{\mu \in \operatorname{Rep}(\hat{\mathfrak{g}})} \mathcal{H}_{\mu} \otimes \overline{\mathcal{H}}_{\mu}^{*}
$$

where $\mathcal{H}_{\mu}$ an $\mathcal{H}_{\mu}^{*}$ are the Kac modules and their duals, as defined in equation (5.1) 22 It should be kept in mind though that $\mathbb{H}$ contains an atypical sector (including, e.g., $\mathcal{H}_{0} \otimes \overline{\mathcal{H}}_{0}^{*}$ ) which is not fully reducible. Nevertheless, the zero-modes $L_{0}$ and $\bar{L}_{0}$ of the Virasoro-Sugawara fields are fully diagonalizable.

The true state space $\mathcal{H}$ of the interacting theory, on the other hand, is a field theoretic version of the space $\mathcal{F}(G)$ in our minisuperspace theory. In particular, $\mathcal{H}$ agrees with $\mathbb{H}$ as a graded vector space (with the grading provided by the generalized eigenvalues of $L_{0}$ and $\bar{L}_{0}$ ) and even as $\hat{\mathfrak{g}}_{0} \oplus \hat{\mathfrak{g}}_{0}$-module. But when considered as a module of the left and/or right current superalgebra, $\mathcal{H}$ and $\mathbb{H}$ are fundamentally different. While, under the action of e.g. the right moving currents, $\mathbb{H}$ decomposes into a sum of typical and atypical Kac modules, $\mathcal{H}$ may be expanded into projectives. The corresponding multiplicity spaces, however, do not carry a representation of the left moving

\footnotetext{
${ }^{21}$ In case the consistency of the bosonic theory requires to consider spectral flow automorphisms, e.g. for noncompact groups, they should also be included in the definition of the labels $\mu$.

${ }^{22}$ It is the dual which is relevant here since we assume the antiholomorphic current superalgebra to mimic the differential operators (4.4), not those in (4.3). Notice that the roles of $S_{1}^{a}$ and $S_{2 a}$ are exchanged in these expressions.
} 
currents, in contrast to what we have seen in eq. (5.20). Instead, atypical representations of the left and right moving currents form large non-chiral modules $\hat{\mathcal{I}}_{[\sigma]}$ which entangle projective covers in an intricate way 23 Now recall that the Virasoro element $L_{0}$ contains the (renormalized) Casimir operator of $\mathfrak{g}$ as a summand and it agrees with the latter on ground states. But since our harmonic analysis revealed that the Casimir operator may not be diagonalized in the atypical subspace of $\mathcal{F}(G)$, the same must be true for the action of $L_{0}$ (and $\bar{L}_{0}$ ) on $\mathcal{H}$. This shows that supergroup WZNW theories are always logarithmic conformal field theories 24

After these remarks, let us address the partition function of the theory and its modular invariance. We have stressed above that $\mathbb{H}$ and $\mathcal{H}$ are isomorphic as $\hat{\mathfrak{g}}_{0} \oplus \hat{\mathfrak{g}}_{0}$-modules. Hence, the partition function of the interacting theory agrees with the partition function of the decoupled model and both may be written as a sum over bilinears of characters of Kac modules 25 Thereby, the partition function of WZNW models on type I supergroups takes the form

$$
Z^{G}(q, \bar{q})=Z_{\mathrm{ren}}^{G_{B}}(q, \bar{q}) \cdot Z_{F}(q, \bar{q})
$$

i.e. it is obtained as a product of the corresponding partition functions of the (renormalized) bosonic model with that of the free fermionic system. Each of the two factors corresponds to a well-defined and consistent conformal field theory. This shows that our proposal for the state space of the supergroup WZNW model yields a suitable partition function.

In theories with fermions one has to distinguish between the purely combinatorial partition function which merely counts states and the torus vacuum amplitude which is the relevant physical quantity. Since the fermions anti-commute, the latter requires an insertion of the fermion number operator $(-1)^{F+F}$ into the trace, thus turning characters into supercharacters. In our state spaces, bosonic and fermionic states always come in pairs, causing $Z_{F}(q, \bar{q})$ to vanish. Actually, this is the usual way in which modular invariance manifests itself in fermionic theories. To avoid dealing with trivial quantities, one may switch to unspecialized characters. The latter lead to a non-vanishing physical partition function.

We claim that the expression (5.21) is the universal partition function for supergroup WZNW models similar to the charge conjugate one in ordinary bosonic models. We will indeed argue in the following section that this modular invariant can be used as the basic building block to derive new, non-trivial partition functions using methods that are well-established in purely bosonic conformal field theories.

We wish to conclude this subsection with a few comments on the calculation of correlation functions. We have argued above that fields in the decoupled and the interacting theory are in one-to-one correspondence with each other. In fact, the transition from the auxiliary space $\mathbb{H}$ to the proper state space $\mathcal{H}$ of the supergroup WZNW model is implemented by a linear map $\hat{\Xi}: \mathbb{H} \rightarrow \mathcal{H}$. The latter generalizes and extends the map $\Xi$ that we used in the semi-classical analysis to identify states in $\mathbb{F}(G)$ and $\mathcal{F}(G)$. Let us denote the image of the field (5.19) under $\hat{\Xi}$ by $\Phi_{\mu ; \mathbf{b}}^{\mathbf{a}}$. According

\footnotetext{
${ }^{23}$ Note that the structure and number of $\hat{\mathfrak{g}}$-blocks and hence of the indecomposables $\hat{\mathcal{I}}_{[\sigma]}$ in the field theory may differ from that in the minisuperspace theory, see eq. (4.7). The relation between the two may be established with the help of spectral flow automorphisms.

${ }^{24}$ There might exist consistent truncations to diagonalizable subsectors for low levels, see the discussion in 37. Such phenomena appear to be very rare, though.

${ }^{25}$ Since the Cartan subalgebra of $\mathfrak{g}$ was assumed to be identical to the Cartan subalgebra of $\mathfrak{g}_{0}$ this statement even holds for unspecialized characters and partition functions.
} 
to our general strategy, correlation functions in the interacting theory may be computed through

$$
\left\langle\Phi_{\mu_{1} ; \mathbf{b}_{1}}^{\mathbf{a}_{1}}\left(z_{1}, \bar{z}_{1}\right) \cdots \Phi_{\mu_{N} ; \mathbf{b}_{N}}^{\mathbf{a}_{N}}\left(z_{N}, \bar{z}_{N}\right)\right\rangle=\sum_{s=0}^{s_{\max }} \frac{1}{s !}\left\langle V_{\mu_{1} ; \mathbf{b}_{1}}^{\mathbf{a}_{1}}\left(z_{1}, \bar{z}_{1}\right) \cdots V_{\mu_{N} ; \mathbf{b}_{N}}^{\mathbf{a}_{N}}\left(z_{N}, \bar{z}_{N}\right) \mathcal{S}_{\text {int }}^{s}\right\rangle_{0}
$$

where the correlators on the right hand side are to be evaluated in the decoupled theory. We shall show below that correlators with $s \geq s_{\max }=N r$ insertions vanish so that the summation over $s$ is finite. Let us also recall that the interaction term is given by

$$
\mathcal{S}_{\text {int }}=-\frac{i}{2 \pi} \int p_{a} R_{b}^{a}\left(g_{B}\right) \bar{p}^{b} d w \wedge d \bar{w}
$$

Here, the expression $R_{b}^{a}\left(g_{B}\right)$ should be interpreted as a vertex operator of the bosonic WZNW model, transforming in the representation $R \otimes R^{*}$.

There are now two computations to be performed in the decoupled theory. First of all, we have to determine correlation functions for the bosonic fields $V_{\mu_{i}}$ with additional insertions of $s$ vertex operators $R_{b}^{a}\left(g_{B}\right)$. We shall assume the bosonic WZNW model to be solved and hence that all these bosonic correlators are known. Let us comment, however, that the dependence of such correlation functions on the insertion points of $R_{b}^{a}\left(g_{B}\right)$ is controlled by null vector decoupling equations. As usual, these can be exploited to derive integral formulas for the required correlation functions. We shall not go into any more detail here.

Instead, let us now comment on the second part of the computation that deals with the fermionic sector. Since we are dealing with $r$ chiral $b c$ systems at central charge $c=-2$, the evaluation is rather standard. According to the usual rules, non-vanishing correlators on the sphere must satisfy $\# \theta^{a}-\# p_{a}=1$, i.e. the number of insertions of a fixed field $\theta^{a}$ must exceed the number of insertions of $p_{a}$ by one. In an $N$-point correlator, any given component $\theta^{a}$ can appear at most $N$ times. The fields $p_{a}$, on the other hand, only emerge from the $s$ insertions of the interaction term. Hence, we conclude that all contributions to our correlation function with $s \geq N \cdot r$ insertions of $\mathcal{S}_{\text {int }}$ vanish. The non-vanishing terms can be evaluated using that

$$
\left\langle\prod_{\nu=1}^{n} p_{a}\left(z_{\nu}\right) \prod_{\mu=1}^{n+1} \theta^{a}\left(x_{\mu}\right)\right\rangle_{0}=\frac{\prod_{\nu<\nu^{\prime}}\left(z_{\nu}-z_{\nu^{\prime}}\right) \prod_{\mu<\mu^{\prime}}\left(x_{\mu}-x_{\mu^{\prime}}\right)}{\prod_{\nu} \prod_{\mu}\left(z_{\nu}-x_{\mu}\right)}
$$

and a similar formula applies to $\bar{\theta}_{a}$ and $\bar{p}^{a}$. These expressions can be inserted into the expansion (5.22). Thereby we obtain a formula for the $N$-point functions of the WZNW model which presents it as a sum of at most $N \cdot r$ terms labeled by an integer $s$. Each individual summand involves an integration over $s$ insertion points $w_{i}$. The corresponding integrand factorizes into free field correlators of the form (5.24) multiplied with a non-trivial $(N+s)$-point function in the bosonic WZNW model for the group $G_{B}$.

Let us point out that for a given choice of $N$ fields, the perturbative evaluation of the correlator may truncate way before we reach $s_{\max }$. An extreme example appears when all the fields $\Phi_{\mu_{i}}=$ $\hat{\Xi} V_{\mu_{i}}, i=2, \ldots, N$, are images of purely bosonic fields $V_{\mu_{i}}$ while the first field contains the maximal number of fermionic factors, both for left and right movers. In that case, only the term with $s=0$ contributes and hence these fields of the WZNW model on the supergroup possess the same correlation functions as in the bosonic WZNW model, i.e.

$$
\left\langle\Phi_{\mu_{1} ; 1,2, \ldots, r}^{1,2, \ldots, r}\left(z_{1}, \bar{z}_{1}\right) \Phi_{\mu_{2}}\left(z_{2}, \bar{z}_{2}\right) \cdots \Phi_{\mu_{N}}\left(z_{N}, \bar{z}_{N}\right)\right\rangle=\left\langle V_{\mu_{1}}\left(z_{1}, \bar{z}_{1}\right) V_{\mu_{2}}\left(z_{2}, \bar{z}_{2}\right) \cdots V_{\mu_{N}}\left(z_{N}, \bar{z}_{N}\right)\right\rangle_{0}
$$


where the correlation function on the right hand side is to be evaluated in the bosonic WZNW model. The result is a direct analogue of the corresponding formula (4.26) in the minisuperspace theory.

\subsection{Some comments on non-trivial modular invariants}

During the course of the previous sections we frequently assumed that the bosonic subgroup $G_{B} \subset G$ was compact and simply-connected. On a technical level, this condition is required in order to render the matrix $R\left(g_{B}\right)$ well-defined which entered the expression for the differential operators implementing the isometries of $G$ on the function space $\mathcal{F}(G)$. On the other hand this choice automatically limited our considerations to WZNW models with (the analogue of a) charge conjugate modular invariant. In this subsection we would like to sketch how such a restriction may be overcome.

Let us recall the situation for bosonic WZNW models first. It is well-known that a non-simplyconnected group manifold $G_{0}$ can be described geometrically as an orbifold $\tilde{G}_{0} / \Gamma$ where $\tilde{G}_{0}$ is the universal covering group and $\Gamma \cong \pi_{1}\left(G_{0}\right) \subset \mathcal{Z}\left(\tilde{G}_{0}\right)$ is a subgroup of its center. The simplest example is $S O(3)=S U(2) / \mathbb{Z}_{2}$. In conformal field theory, orbifolds of the previous type are implemented by means of a simple current extension of the theory with charge conjugate modular invariant 65] (see also [66]). This construction of the $G_{0}$ WZNW model rests on the fact that the $\tilde{G}_{0}$ model contains sufficiently many simple currents, one for each element in the center $\mathcal{Z}\left(\tilde{G}_{0}\right)$. Incidently, these are in one-to-one correspondence with (spectral flow) automorphisms of the current algebra $\hat{\mathfrak{g}}_{0}$. Such simple current extensions exhaust all modular invariants related to the current algebra $\hat{\mathfrak{g}}_{0}$, apart from some exceptional cases at low levels.

Now it has been shown in [67] that the global topology of a Lie supergroup is completely inherited from that of its bosonic subgroup. Consequently, given a supergroup $G$ with bosonic subgroup $G_{0}=\tilde{G}_{0} / \Gamma$, there exists a covering supergroup $\tilde{G}$ with bosonic subgroup $\tilde{G}_{0}$, and one has $G=\tilde{G} / \Gamma$. Note that central elements in $\tilde{G}_{0}$ are also central in $\tilde{G}$. Having constructed the WZNW model on the covering supergroup $\tilde{G}$, we would like to divide by $\Gamma$. But, as we have just stated, elements of $\Gamma$ can all be identified with elements in the center of the bosonic subgroup $\tilde{G}_{0}$. Therefore, they label certain simple currents of the $\tilde{G}_{0}$ WZNW model. As indicated in the previous paragraph, we may think of these simple currents as (equivalence classes of) spectral flow automorphisms of $\hat{\mathfrak{g}}_{0}$. According to the results of subsection 5.2, all such spectral flow automorphisms may be extended from $\hat{\mathfrak{g}}_{0}$ to the current Lie superalgebra $\hat{\mathfrak{g}}$, in a way that is even consistent with the free fermion construction. Consequently, the elements of our designated orbifold group $\Gamma$ label a certain set of spectral flow automorphisms of $\hat{\mathfrak{g}}$. It is the action of these spectral flow automorphisms that one has to use in order to construct the orbifold CFT belonging to the supergroup $G=\tilde{G} / \Gamma$.

Our discussion so far has been fairly abstract and we would like to flesh it out a bit more. Actually, the details of the orbifold construction are not much different from what is done in bosonic models. For simplicity, let us assume that $\Gamma$ is cyclic and of finite order. We shall denote the generating element by $\gamma$. In order to illustrate the relation between orbifolds and spectral flow automorphisms, we depart from the conventional orbifold approach. Namely, we include (chiral) twisted sectors on which the supercurrents $X$ satisfy boundary conditions of the form

$$
X\left(e^{2 \pi i} z\right)=(\gamma(X))(z) .
$$

There exists a basis $X_{\sigma}$, on which $\gamma$ acts diagonally as a multiplication with some phase $\exp \left(2 \pi i \gamma_{\sigma}\right)$. If $\gamma_{\sigma}$ is an integer, then $X_{\sigma}$ has integer moding in the twisted sector, otherwise its modes are 
rational. All these twisted sectors emerge by acting with certain (meromorphic or not) spectral flow automorphisms on the untwisted representations (see subsection 5.2). The discussion of the previous paragraph supplied us with the relevant set of spectral flow automorphisms and hence with a list of chiral sectors to be incorporated in the construction of the $G=\tilde{G} / \Gamma$ orbifold theory. Sectors of the full non-chiral theory are obtained by independent action of spectral flow automorphisms on left and right-movers in the parent theory on $\tilde{G}$. Therefore, even meromorphic spectral flows lead to new non-chiral sectors, though these are put together from untwisted representations of the left and right movers. All this has been worked out for many interesting bosonic models, such as e.g. the $S O(3)=S U(2) / \mathbb{Z}_{2}$ WZNW model. WZNW models on non-simply-connected supergroups are no harder to deal with 26

Let us finally comment on the connection of the algebraic orbifolds with the Lagrangian picture. Looking at our free field resolution (3.5) one might have had the naive idea to replace the bosonic model by its orbifold and then to add fermions and interaction terms in the same way as before. But this is not at all what we suggest to do. In particular, the orbifold group $\Gamma$ need not be a symmetry of the interaction term if there is no action on the fermions. Even worse, the vertex operator $R_{b}^{a}\left(g_{B}\right)$ occuring in the interaction may not be part of the spectrum of the purely bosonic WZNW model. As a consequence, the perturbed correlation functions with insertions of this operator are not well-defined. This happens, for example, if we try to supersymmetrize the bosonic group $S O(3) \times U(1)$. The fermions of the extended model with $s u(2 \mid 1)$ symmetry transform in the spin $1 / 2$ representation of $S U(2)$ which does not descend to a representation of $S O(3)=S U(2) / \mathbb{Z}_{2}$. Hence, it is absolutely crucial to depart from the full $S U(2 \mid 1)$ WZNW model and to divide the full orbifold action on both bosonic and fermionic variables.

\section{Lessons for other logarithmic CFTs}

Various logarithmic conformal field theories have been considered in the literature. The best studied examples are the triplet models in which the conformal symmetry is extended by a triplet of currents, each having spin $h=2 p-1$ [68]. For most of these algebras only chiral aspects have been investigated so far, but in case of $p=2$, Gaberdiel and Kausch have been able to come up with a consistent local theory [11. The extended chiral symmetry of the triplet models is denoted by $\mathcal{W}_{1, p}$. The latter are believed to be part of a family of more general $\mathcal{W}$-algebras $\mathcal{W}_{q, p}$ where $p$ and $q$ are co-prime. All of these possess interesting indecomposable representations. Their representation theory is particularly well understood for $q=1$, see [14] and references therein.

This final section has two aims. First of all we would like to illustrate that the existing results on the representation theory of $\mathcal{W}_{1, p^{-}}$algebras and the local triplet model (for $p=2$ ) fit very nicely into one common picture with the logarithmic WZNW models on type I supergroups. But given the remarkable progress with the latter, and in particular with the construction of infinitely many families of new local non-chiral models, our results lead to a number of interesting predictions on $\mathcal{W}_{q, p^{-}}$algebras and the associated local logarithmic conformal field theories.

${ }^{26}$ The $S O(3)$ theory also shows that the orbifold construction might suffer from obstructions, depending on the choice of the level. A more detailed treatment of such issues for supergroup orbifolds is left for future work. 


\subsection{Chiral representation theory}

Let us begin this subsection by reviewing some results on the representation theory of $\mathcal{W}_{1, p}=\mathcal{W}(p)$ (see [14] and references therein). This chiral algebra is known to admit $2 p$ irreducible highest weight representations $\mathcal{V}_{s}^{ \pm}$where $s=1, \ldots, p$. While $\mathcal{V}_{p}^{ \pm}$do not admit non-split extensions, all other $2(p-1)$ representations appear in the head of the following indecomposables, 27

$$
\mathcal{R}_{s}^{ \pm}: \quad \mathcal{V}_{s}^{ \pm} \rightarrow 2 \mathcal{V}_{p-s}^{\mp} \rightarrow \mathcal{V}_{s}^{ \pm}
$$

where $s$ runs from $s=1$ to $s=p-1$. Hence the representations $\mathcal{V}_{p}^{ \pm}$can be considered typical whereas all others are atypical 28 Moreover, the indecomposables $\mathcal{R}_{s}^{ \pm}$are the projective covers of the atypicals $\mathcal{V}_{s}^{ \pm}$and play the role of the representations $\mathcal{P}$ in section 2.2.3. The typical modules $\mathcal{V}_{p}^{ \pm}$are projective as well, in agreement with results on the fusion for $\mathcal{W}(2)$ representations, see [69]. The fusion rules of $\mathcal{W}_{p, q}$-models have recently been addressed in [16].

The representation theory of $\mathcal{W}(p)$-algebras also contains analogues of our Kac modules for atypical representations. These have the form

$$
\mathcal{K}_{s}^{ \pm}: \mathcal{V}_{s}^{ \pm} \rightarrow \mathcal{V}_{p-s}^{\mp}
$$

where $s=1, \ldots, p-1$. In view of the role they are going to play we will simply refer to the representations $\mathcal{K}_{s}^{ \pm}$as "Kac modules" as well 29 They are obtained as quotients of the projective covers $\mathcal{R}_{s}^{ \pm}$. For the typical representations $\mathcal{V}_{p}^{ \pm}$, the associated irreducibles, "Kac modules" and projective covers all coincide. In this sense, we shall also write $\mathcal{K}_{p}^{ \pm}=\mathcal{V}_{p}^{ \pm}=\mathcal{R}_{p}^{ \pm}$, just as for typical representations of Lie superalgebras. Furthermore, among the quotients of the projective covers one can also find $4(p-1)$ "zig-zag" modules, containing three irreducible representations each. It seems likely, that these are just the first few examples among an infinite series of zig-zag representations of $\mathcal{W}(p)$, in close analogy to representations of the Lie superalgebra $g l(1 \mid 1)$ (see e.g. [71]). The main difference between $g l(1 \mid 1)$ and $\mathcal{W}(p)$ zig-zag modules is that the constituents of the former are pairwise inequivalent. Zig-zag modules of $\mathcal{W}(p)$, on the other hand, are built from a pair of irreducibles, each appearing with some multiplicity. This opens the possibility to close zig-zag modules of $\mathcal{W}(p)$ into rings. Representations of all these different shapes were found and investigated for the quantum groups [72, 73] which are dual to $\mathcal{W}(p)$, in the sense of KazhdanLusztig duality.

Let us also compare some further properties of $\mathcal{W}(p)$-modules with those we discussed for Lie superalgebras of type I. For example, we have pointed out that all projective modules of type I superalgebras possess a "Kac composition series". The same is true for the projective covers $\mathcal{R}_{s}^{ \pm}$,

$$
\mathcal{R}_{s}^{ \pm}: \quad \mathcal{K}_{s}^{ \pm} \rightarrow \mathcal{K}_{p-s}^{\mp} \quad(\text { for } s<p) \quad, \quad \mathcal{R}_{p}^{ \pm}=\mathcal{K}_{p}^{ \pm} .
$$

Moreover, we also observe that the multiplicities in the "Kac composition series" of indecomposable projective covers (reducible and irreducible) and those of irreducible representations in the composition series of "Kac modules" are related by

$$
\left(\mathcal{R}_{\mu}: \mathcal{K}_{\nu}\right)=\left[\mathcal{K}_{\nu}: \mathcal{V}_{\mu}\right]
$$

\footnotetext{
${ }^{27}$ These diagrams have to be read as follows: To the right we write the maximal fully reducible submodule. Everything left of the rightmost arrow describes the quotient module of the original module with respect to the submodule mentioned before. One can then proceed iteratively to define the whole diagram.

${ }^{28}$ We use the qualifiers "atypical" and "typical" only to clarify the analogy to the supergroup WZNW models. In contrast to the latter, the atypical representations are obviously the generic ones for the algebra $\mathcal{W}(p)$.

${ }^{29}$ Using the analogy to the Kazhdan-Lusztig dual quantum group, they have been called Verma modules in [70].
} 
This establishes an analogue of the reciprocity theorem (2.11) that has been an important ingredient in our description of supergroup WZNW models and, in particular, in exhibiting its modular invariance.

The agreement between algebraic structures in the representation theory of Lie superalgebras and of symmetries in minimal logarithmic conformal field theories is remarkable. But let us think ahead and see what Lie superalgebras may teach us for future studies of indecomposable $\mathcal{W}$ algebra representations. While irreducible representations and their projective covers are certainly central objects for all Lie superalgebras, some of their properties may differ considerably from what we have seen in the case of type I. We have pointed out already that the existence of a "Kac composition series" (or a similar flag) for projectives and the reciprocity property (2.11) do not hold for more general Lie superalgebras. Hence, these features of $\mathcal{W}(p)$-modules should not be expected to carry over to more general $\mathcal{W}$-algebras either. In fact, numerical results of [74] may indicate that violations even occur for $\mathcal{W}_{q, p}$ with $p, q \neq 1$. Furthermore, the tensor products for irreducible representations of Lie superalgebras can develop a remarkable complexity. In this sense, the Lie superalgebra $g l(1 \mid 1)$ is rather well-behaved. Representations of $\operatorname{psl}(2 \mid 2)$, for example, are much less tame. In particular, tensor powers of its adjoint representation lead to an infinite series of indecomposables (see [71] for details). The similarities between representations of $g l(1 \mid 1)$ and $\mathcal{W}_{1, p}$ suggest that the latter may also be rather unusual creatures in the zoo of $\mathcal{W}$-algebras. In fact, when it comes to the features of fusion, the algebras $\mathcal{W}_{q, p}$ may have much more generic properties, resembling very closely those of $p s l(2 \mid 2) 30$

\subsection{Local logarithmic conformal field theories}

Regarding the construction of local field theories, the progress with WZNW models on supergroups has been significantly faster than for minimal logarithmic CFTs. In fact, only the minimal triplet model associated with $\mathcal{W}(2)$ has been constructed in all detail [11. Imposing locality constraints on correlation functions, the state space $\mathcal{H}$ of this model was shown to have the form

$$
\mathcal{H}=\mathcal{I}_{1} \oplus\left(\mathcal{V}_{2}^{+} \otimes \overline{\mathcal{V}}_{2}^{+}\right) \oplus\left(\mathcal{V}_{2}^{-} \otimes \overline{\mathcal{V}}_{2}^{-}\right)
$$

Here, $\mathcal{V}_{2}^{ \pm}$are the typical modules of $\mathcal{W}(2)$, in view of their conformal dimensions previously also denoted by $\mathcal{V}_{-1 / 8}$ and $\mathcal{V}_{3 / 8}$, and $\mathcal{I}_{1}$ is a complicated non-chiral indecomposable (denoted by $\mathcal{R}$ in [11]) which was obtained originally as a certain quotient of the space $\left(\mathcal{R}_{1}^{+} \otimes \overline{\mathcal{R}}_{1}^{+}\right) \oplus\left(\mathcal{R}_{1}^{-} \otimes \overline{\mathcal{R}}_{1}^{-}\right)$. The module $\mathcal{I}_{1}$ is known to possess the following composition series

$$
\mathcal{I}_{1}: \quad\left(\mathcal{V}_{1}^{+} \otimes \overline{\mathcal{V}}_{1}^{+}\right) \oplus\left(\mathcal{V}_{1}^{-} \otimes \overline{\mathcal{V}}_{1}^{-}\right) \rightarrow 2\left(\mathcal{V}_{1}^{+} \otimes \overline{\mathcal{V}}_{1}^{-}\right) \oplus 2\left(\mathcal{V}_{1}^{-} \otimes \overline{\mathcal{V}}_{1}^{+}\right) \rightarrow\left(\mathcal{V}_{1}^{+} \otimes \overline{\mathcal{V}}_{1}^{+}\right) \oplus\left(\mathcal{V}_{1}^{-} \otimes \mathcal{V}_{1}^{-}\right)
$$

where we used the correspondence $\mathcal{V}_{1}^{+}=\mathcal{V}_{0}$ and $\mathcal{V}_{1}^{-}=\mathcal{V}_{1}$ for the atypical irreducibles of $\mathcal{W}(2)$ When acting with elements of either the left or right chiral algebra only, $\mathcal{H}$ decomposes into a sum of projectives, each appearing with infinite multiplicity. The individual multiplicity spaces cannot be promoted to representation spaces of the commuting chiral algebra, but they come equipped with a grading that is given by the (generalized) eigenvalues of $L_{0}$ or $\bar{L}_{0}$. When considered as graded vector spaces, they coincide with the graded carrier spaces of irreducible representations. All this is very reminiscent of what we found in eq. (4.6) while studying the harmonic analysis on supergroups.

\footnotetext{
${ }^{30} \mathrm{~A}$ certain similarity between the representation theory of $\mathcal{W}_{q, p}$ (or rather its dual quantum group) and $p s l(2 \mid 2)$ is suggested by the structure of their respective projective covers, cf. e.g. Figure 7 of [75] with eq. (2.12) of [76].
} 
Carrying on with the comparison between the triplet model and WZNW models on supergroups, we also observe that the composition series of the state space (6.5) agrees with that of the module $\left(\mathcal{K}_{1}^{+} \otimes \overline{\mathcal{K}}_{1}^{+}\right) \oplus\left(\mathcal{K}_{1}^{-} \otimes \overline{\mathcal{K}}_{1}^{-}\right)$. Hence, the partition function of the triplet model can be expressed as

$$
Z(q, \bar{q})=\sum_{i=1,2} \sum_{\eta= \pm} \chi_{\mathcal{K}_{i}^{\eta}}(q) \bar{\chi}_{\mathcal{K}_{i}^{\eta}}(\bar{q})
$$

This result is reminiscent of what we found for supergroup WZNW models in section 5.3. Note that the modular transformation behaviour for characters of Kac modules is rather simple which makes it easy to check that $Z(q, \bar{q})$ is modular invariant. In comparison, the transformation behaviour of characters belonging to atypical irreducible representations of $\mathcal{W}(2)$ is rather involved [69], just as for current superalgebras.

The striking similarities between the local triplet theory and the harmonic analysis on supergroups suggest some far reaching generalizations, in particular concerning the state space of a wide class of local logarithmic conformal field theories. Let us denote the irreducible representations of some chiral algebra $\mathcal{W}$ by $\mathcal{V}_{a}$ and their projective covers by $\mathcal{P}_{a}$. For typical representations the latter agree (by definition) with the irreducibles. We also introduce the symbol $V_{a}$ when $\mathcal{V}_{a}$ is considered merely as an $L_{0}$-graded vector space. Given this notation, we propose that a local logarithmic conformal field theory with symmetry $\mathcal{W}$ can be constructed on the state space

$$
\mathcal{H}=\bigoplus_{a} V_{a} \otimes \overline{\mathcal{P}}_{a}
$$

Our proposal describes the state space of the conjectured local theory as a graded representation space for $\mathcal{W}$. The extension to the full $\mathcal{W} \otimes \overline{\mathcal{W}}$ is severely constrained by requiring symmetry with respect to an exchange of left and right chiral algebras. Concerning the implications for $\mathcal{W}(p)$ models it is interesting to observe that the same structures were found in the regular representation of the dual quantum group, see [70, page 24, and compare with eq. (2.9) in [37]. Let us point out that local theories may probably also be built on other state spaces. Examples are given by the orbifold models we described in section 5.4 or by some exceptional truncations of WZNW models on simply connected Lie supergroups (see [37] for a few examples).

Before we conclude we would like to go one step beyond the previous analogy and to propose a more detailed conjecture for the natural state space of the $\mathcal{W}(p)$ triplet models for arbitrary $p$. In a straightforward extension of the result (6.5) for $p=2$ we believe that a local theory may be built on the space

$$
\mathcal{H}=\bigoplus_{s \neq p} \mathcal{I}_{s} \oplus \bigoplus_{\eta= \pm} \mathcal{V}_{p}^{\eta} \otimes \overline{\mathcal{V}}_{p}^{\eta}
$$

The non-chiral indecomposable representations occurring here have the composition series

$\mathcal{I}_{s}: \quad\left(\mathcal{V}_{s}^{+} \otimes \overline{\mathcal{V}}_{s}^{+}\right) \oplus\left(\mathcal{V}_{p-s}^{-} \otimes \overline{\mathcal{V}}_{p-s}^{-}\right) \rightarrow 2\left(\mathcal{V}_{s}^{+} \otimes \overline{\mathcal{V}}_{p-s}^{-}\right) \oplus 2\left(\mathcal{V}_{p-s}^{-} \otimes \overline{\mathcal{V}}_{s}^{+}\right) \rightarrow\left(\mathcal{V}_{p-s}^{-} \otimes \overline{\mathcal{V}}_{p-s}^{-}\right) \oplus\left(\mathcal{V}_{s}^{+} \otimes \mathcal{V}_{s}^{+}\right)$

which coincides with the composition series of $\left(\mathcal{K}_{s}^{+} \otimes \mathcal{K}_{s}^{+}\right) \oplus\left(\mathcal{K}_{p-s}^{-} \otimes \mathcal{K}_{p-s}^{-}\right)$. Consequently, our proposal is manifestly modular invariant since the partition function can be written as a sum over all "Kac modules", just as in eq. (6.7). Figure 1 provides an alternative 2-dimensional picture of the indecomposables $\mathcal{I}_{s}$. In this form the similarities with analogous pictures for $g l(1 \mid 1)$ and $\operatorname{sl}(2 \mid 1)$ [36, 37] and for the quantum group dual of $\mathcal{W}(p)$-models [70] are clearly displayed. 


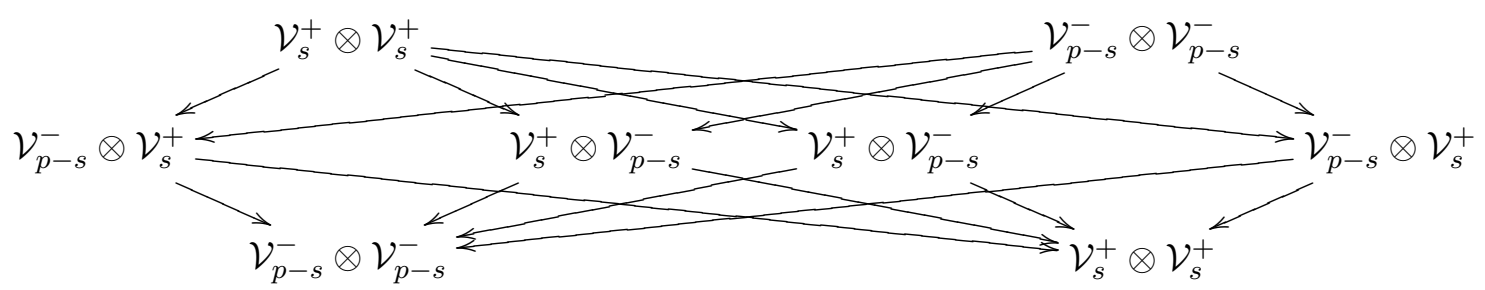

Figure 1: The structure of the non-chiral representation $\mathcal{I}_{s}$.

The particular relevance of projective modules for local bulk theories is one of the main outcomes from the study of WZNW models on supergroups, see also [36, 24, 37]. Their role for logarithmic extensions of minimal models was also emphasized in [75, 77, mostly based on studies of the dual quantum group. It seems worth pointing out, though, that for quotients of supergroups, projective modules might not play such a prominent role, even though some of them are likely to be logarithmic as well. Similarly, boundary spectra in logarithmic conformal field theories are known to involve atypical irreducibles as well as projectives. For the triplet model, boundary conditions with an atypical irreducible spectrum of boundary operators were exhibited in the recent work of Gaberdiel and Runkel [18. Studies of branes on supergroups confirm the existence of such boundary spectra and they provide a beautiful geometric explanation [78].

\section{Outlook and open questions}

In our paper we presented the main ingredients for a complete solution of arbitrary supergroup WZNW models based on basic Lie superalgebras of type I. All our results relied on a free fermion resolution of the underlying current superalgebra which allowed to keep the bosonic subsymmetry manifest in all expressions we encountered, i.e. in action functionals, representations, correlation functions and other quantities. On the level of the Lagrangian we showed that the original WZNW Lagrangian could be written as a sum of a WZNW model for the bosonic subgroup with renormalized metric and possibly a dilaton, the action for a set of free fermions and an interaction term which couples the fermions to a vertex operator of the bosonic model. The usefulness of this construction has also been demonstrated in the full quantum theory, e.g. when we reconstructed the current superalgebra in terms of the corresponding bosonic current algebra and free fermions.

In order to solve the WZNW model we first focused on its semi-classical, or small curvature limit which allowed to reduce the construction of the space of ground states to a problem in harmonic analysis on a supergroup. We could confirm previous observations [58, 36, 24, 37] that the space of functions splits into two qualitative very different sectors. First of all, there exists a typical sector which decomposes into a tensor product of irreducible typical representations under the action of the supergroup isometry $\mathfrak{g} \oplus \mathfrak{g}$. On this subspace, the Laplacian is fully diagonalizable and its eigenvalues are determined by a specific quadratic Casimir of $\mathfrak{g}$. In addition, the space of functions on a supergroup always exhibits an atypical sector consisting of projective covers entangled in a complicated way such that the resulting non-chiral modules cannot be written as (a direct sum of) tensor product representations. In this sector the Laplacian is not diagonalizable and the necessity for a non-trivial entanglement may eventually be traced back to the fact that the left and right 
regular action lead to the same expression for the Laplacian. We wish to emphasize that our derivation of the spectrum has been very general and just relied on the validity of a reciprocity theorem proven by Zou and Brundan [48, 49].

Starting from this semi-classical truncation it has been argued that all its interesting features persist in the full quantum theory. In particular, the full state space of the WZNW model is still composed of a typical and an atypical sector. Again, the representations in the latter do not factorize and the dilatation operators $L_{0}$ and $\bar{L}_{0}$ may not be diagonalized. Since the vacuum representation is always atypical this automatically implies the existence of a logarithmic partner of the identity field and makes supergroup WZNW models genuine examples of logarithmic conformal field theories.

It should be noted that, in comparison to ordinary free field constructions [38, 39, 40, 41, 42] which are based on a choice of an abelian subalgebra, our free fermion resolution is much easier to deal with. In particular, the representations of the current superalgebra obtained from the generalized Fock spaces (5.1) are typically irreducible. This observation lets us suggest that these representations are the proper generalization of Kac modules in the infinite dimensional setting. Furthermore, there was no need of introducing various screening charges and BRST operators, a simplifying feature that reflects itself in the calculation of correlation functions. The latter could be reduced to a perturbative but finite expansion in terms of correlation functions in the product of a purely bosonic WZNW model with renormalized metric and a theory of free fermions.

Finally, we commented on possible partition functions and we explained how they are constructed as a product of partition functions for the constituents in our free fermion resolution. This rather simple behavior is rooted in the fact that traces are insensitive to the composition structure of representations. Hence, the full WZNW theory possesses the same partition function as the decoupled free fermion theory in which products of (reducible) Kac modules appear instead of projective covers. Taking this assertion for granted, the torus modular invariance of our theory is satisfied automatically. It might be helpful to add that torus partition functions of many nonrational bosonic conformal field theories, e.g. of Liouville theory or of the $H_{+}^{3}$ model, are equally insensitive to the interaction. This does certainly not imply that the theories are trivial, neither in case of non-rational conformal field theories, nor for WZNW models on supergroups.

In the last section of this work we placed our new results on chiral and non-chiral aspects of supergroup WZNW models in the context of previous and ongoing work on other logarithmic conformal field theories, in particular on logarithmic extensions of minimal models. The similarities are remarkable and provide some novel insight that helps to separate generic properties of logarithmic conformal field theories from rather singular coincidences. As an application of the analogies we conjectured a precise formula for the state space of a fully consistent local theory based on an arbitrary chiral algebra. It adopts a particularly nice shape for the minimal logarithmic $\mathcal{W}(p)$-theories.

Working with supergroup WZNW models has two important advantages over the consideration of non-geometric logarithmic conformal field theories. Concerning the study of chiral aspects, the close link between the current superalgebra $\hat{\mathfrak{g}}$ and its horizontal subsuperalgebra $\mathfrak{g}$ provides a rather strong handle on the representation theory of $\mathcal{W}=\hat{\mathfrak{g}}$. In fact, since the representation theory of $\mathfrak{g}$ is under good control, the same is true for its affine extension $\hat{\mathfrak{g}}$. Even though we have not really pushed this to the level of mathematical theorems, there is no doubt that rigorous results can be established along the lines of our discussion. For some particular examples, this has been carried out already [36, 24, 37]. The second advantage of supergroup WZNW models is the existence of an action principle. The latter is particularly powerful when it comes to the construction of 
local logarithmic field theories, a subject that has been notoriously hard to address for logarithmic extensions of minimal models. In fact, we have seen in section 5.3 that the action leads to a rigorous tool for constructing bulk correlation functions. As such, it has already been exploited in the construction of correlation functions for the $G L(1 \mid 1)$ WZNW model [36].

The present work admits natural extensions in several directions. Among these, the problem of finding concrete expressions for the full correlation functions or, at least, conformal blocks is probably the most urgent. Another issue of considerable significance is the extension of our ideas to world-sheets with boundaries or, in string theory language, the discussion of D-branes. In this context it seems necessary to obtain a better handle on modular transformation properties of characters, including those of irreducible atypical representations [13]. We hope that our work will be helpful in deriving new character formulas along the lines of [24, 37]. It would also be interesting to work out in greater detail the solution of WZNW models with non-trivial modular invariants.

In order to acquire more experience with supersymmetric $\sigma$-models and for various applications it would be desirable to extend our study to supercoset models. In contrast to bosonic models, there is considerably more freedom in choosing how to gauge. Besides gauging the standard adjoint action, as is done in [79, 80, 81, 82, there are many cases in which purely one-sided cosets are known or believed to be conformally invariant [19, 20, 21, 22]. Those latter cases are relevant for the description of AdS-spaces, projective superspaces and even flat Minkowski space. It is worth noting that the harmonic analysis on coset models $G / H$ with $H$ a bosonic subgroup acting from the right, $g \sim g h$, can easily be obtained from our results, see section 4.2 and especially eq. (4.8) (cmp. also [83]). All the additional input required is the branching of $\mathfrak{g}_{0}$-modules into $\mathfrak{h}$-modules. Even before carrying out any such decomposition explicitly, we may conclude from eq. (4.8) that the resulting $\mathfrak{g}$-modules are all projective. This particularly applies to all generalized symmetric spaces which are relevant for the description of AdS-spaces. Let us stress, however, that cosets $G / H$ by some non-trivial supergroup $H$ may behave differently. In fact, some simple examples show how even atypical irreducibles may emerge in their spectrum.

Apart from these structural and conceptual issues we also expect our work to have concrete implications, e.g. in string theory. Let us recall that it is not difficult to write down classical $\sigma$ models which can be used to describe string theory on AdS-spaces with various types of background fluxes for instance [19, 84]. But for a long time it has not been clear how to quantize these field theories while keeping the target space supersymmetry manifest. It was only recently that the pure spinor approach closed this gap to some extent [85, 86. Although substantial progress has been made on certain aspects of the pure spinor formulation, there exist a variety of open conceptual issues, in particular when curved backgrounds are involved. It was proposed to overcome some of them through a reformulation in terms of supergroup WZNW models [87]. The ideas presented above may help to gain more control over the relevant models.

For a complete picture we also need to solve WZNW models beyond Lie supergroups of type I. These include, in particular, supergroups of type II where the fermions occur in a single multiplet of the bosonic subgroup. Structurally, type II implies that there is no natural $\mathbb{Z}$-grading anymore which is consistent with the intrinsic $\mathbb{Z}_{2}$-grading of the underlying Lie superalgebra. This issue concerns the two series $B(m, n)=\operatorname{osp}(2 m+1 \mid 2 n)$ and $D(m, n)=\operatorname{ssp}(2 m \mid 2 n)$ of Kac' classification [45] which e.g. constitute the isometries of superspheres $S^{2 n+m-1 \mid 2 n}$ [5, 88]. Moreover, these series include the special cases $D(2,1 ; \alpha)$ and $D(n+1, n)$ which have been shown [22] to have similarly exciting properties as $A(n, n)=\operatorname{psl}(n+1 \mid n+1)$ [23. Let us note that the WZNW models based on the family of exceptional Lie superalgebras $D(2,1 ; \alpha)$ are also relevant for a manifestly supersymmetric 
description of string backgrounds involving $A d S_{3} \times S^{3} \times S^{3}$. Since type II superalgebras do not admit a canonical (covariant) split of fermionic coordinates into holomorphic and antiholomorphic degrees of freedom, they require a rather significant extension of the above analysis. Incidently, the same is true for the representation theory of type II superalgebras which is considerably more complicated than in the type I case [50]. We hope to return to these issues in future work.

\section{Acknowledgements}

It is a great pleasure to thank Nathan Berkovits, Thomas Creutzig, Matthias Gaberdiel, Gerhard Götz, Andreas Ludwig, Eric Opdam, Jørgen Rasmussen, Alice Rogers, Ingo Runkel, Hubert Saleur, Alexei Semikhatov, Vera Serganova, Arvind Subramaniam and Anne Taormina for useful and inspiring discussions. Thomas Quella acknowledges the warm hospitality at the KITP in Santa Barbara during an intermediate stage of this work. This research was supported in part by the EU Research Training Network grants "Euclid" (contract number HPRN-CT-2002-00325), "ForcesUniverse" (contract number MRTN-CT-2004-005104), "Superstring Theory" (contract number MRTN-CT-2004-512194), by the PPARC rolling grant PP/C507145/1, and by the National Science Foundation under Grant no. PHY99-07949. Part of this work has been performed while Thomas Quella was working at King's College London, funded by a PPARC postdoctoral fellowship under reference PPA/P/S/2002/00370.

\section{References}

[1] G. Parisi and N. Sourlas, Random magnetic fields, supersymmetry and negative dimensions, Phys. Rev. Lett. 43 (1979) 744.

[2] G. Parisi and N. Sourlas, Supersymmetric field theories and stochastic differential equations, Nucl. Phys. B206 (1982) 321.

[3] K. B. Efetov, Supersymmetry and theory of disordered metals, Adv. in Phys. 32 (1983), no. 1 $53-127$.

[4] D. Bernard, (Perturbed) conformal field theory applied to 2D disordered systems: An introduction, hep-th/9509137.

[5] N. Read and H. Saleur, Exact spectra of conformal supersymmetric nonlinear sigma models in two dimensions, Nucl. Phys. B613 (2001) 409 hep-th/0106124.

[6] I. A. Gruzberg, A. W. W. Ludwig and N. Read, Exact exponents for the spin quantum Hall transition, Phys. Rev. Lett. 82 (1999) 4524 cond-mat/9902063.

[7] F. H. L. Essler, H. Frahm and H. Saleur, Continuum limit of the integrable sl(2|1) $3-\overline{3}$ superspin chain, Nucl. Phys. B712 (2005) 513-572 [cond-mat/0501197.

[8] M. R. Zirnbauer, Conformal field theory of the integer quantum Hall plateau transition, hep-th/9905054.

[9] M. J. Bhaseen, I. I. Kogan, O. A. Solovev, N. Tanigichi and A. M. Tsvelik, Towards a field theory of the plateau transitions in the Integer Quantum Hall Effect, Nucl. Phys. B580 (2000) 688-720 cond-mat/9912060. 
[10] A. M. Tsvelik, Evidence for the PSL(2|2) Wess-Zumino-Novikov-Witten model as a model for the plateau transition in quantum Hall effect: Evaluation of numerical simulations, cond-mat/0702611.

[11] M. R. Gaberdiel and H. G. Kausch, A local logarithmic conformal field theory, Nucl. Phys. B538 (1999) 631-658 hep-th/9807091.

[12] M. A. I. Flohr, On modular invariant partition functions of conformal field theories with logarithmic operators, Int. J. Mod. Phys. A11 (1996) 4147-4172 hep-th/9509166.

[13] A. M. Semikhatov, A. Taormina and I. Y. Tipunin, Higher level Appell functions, modular transformations, and characters, Comm. Math. Phys. 255 (2005) 469-512 math.qa/0311314.

[14] J. Fuchs, S. Hwang, A. M. Semikhatov and I. Y. Tipunin, Nonsemisimple fusion algebras and the Verlinde formula, Commun. Math. Phys. 247 (2004) 713-742 hep-th/0306274.

[15] J. Fuchs, On non-semisimple fusion rules and tensor categories, hep-th/0602051.

[16] M. Flohr and H. Knuth, On Verlinde-like formulas in $c_{p, 1}$ logarithmic conformal field theories, arXiv:0705.0545 [math-ph].

[17] S. Kawai and J. F. Wheater, Modular transformation and boundary states in logarithmic conformal field theory, Phys. Lett. B508 (2001) 203 [hep-th/0103197].

[18] M. R. Gaberdiel and I. Runkel, The logarithmic triplet theory with boundary, J. Phys. A39 (2006) 14745-14780 hep-th/0608184.

[19] R. R. Metsaev and A. A. Tseytlin, Type IIB superstring action in $A d S_{5} \times S^{5}$ background, Nucl. Phys. B533 (1998) 109-126 hep-th/9805028.

[20] N. Berkovits, M. Bershadsky, T. Hauer, S. Zhukov and B. Zwiebach, Superstring theory on $A d S_{2} \times S^{2}$ as a coset supermanifold, Nucl. Phys. B567 (2000) 61-86 hep-th/9907200.

[21] D. Kagan and C. A. S. Young, Conformal sigma-models on supercoset targets, Nucl. Phys. B745 (2006) 109-122 hep-th/0512250.

[22] A. Babichenko, Conformal invariance and quantum integrability of sigma models on symmetric superspaces, Phys. Lett. B648 (2007) 254-261 hep-th/0611214.

[23] M. Bershadsky, S. Zhukov and A. Vaintrob, $P S L(n \mid n)$ sigma model as a conformal field theory, Nucl. Phys. B559 (1999) 205-234 hep-th/9902180.

[24] G. Götz, T. Quella and V. Schomerus, The WZNW model on PSU(1,1|2), JHEP 03 (2007) 003 hep-th/0610070].

[25] I. Bena, J. Polchinski and R. Roiban, Hidden symmetries of the $A d S_{5} \times S^{5}$ superstring, Phys. Rev. D69 (2004) 046002 hep-th/0305116.

[26] C. A. S. Young, Non-local charges, $Z_{m}$ gradings and coset space actions, Phys. Lett. B632 (2006) 559-565 hep-th/0503008. 
[27] V. Gurarie, Logarithmic operators in conformal field theory, Nucl. Phys. B410 (1993) 535-549 hep-th/9303160.

[28] H. G. Kausch, Symplectic fermions, Nucl. Phys. B583 (2000) 513-541 hep-th/0003029.

[29] M. R. Gaberdiel, An algebraic approach to logarithmic conformal field theory, Int. J. Mod. Phys. A18 (2003) 4593-4638 hep-th/0111260.

[30] M. Flohr, Bits and pieces in logarithmic conformal field theory, Int. J. Mod. Phys. A18 (2003) 4497-4592 hep-th/0111228.

[31] L. Rozansky and H. Saleur, Quantum field theory for the multivariable Alexander-Conway polynomial, Nucl. Phys. B376 (1992) 461-509.

[32] L. Rozansky and H. Saleur, $S$ and T matrices for the U(1|1) WZW model: Application to surgery and three manifolds invariants based on the Alexander-Conway polynomial, Nucl. Phys. B389 (1993) 365-423 hep-th/9203069.

[33] Z. Maassarani and D. Serban, Non-unitary conformal field theory and logarithmic operators for disordered systems, Nucl. Phys. B489 (1997) 603-625 hep-th/9605062.

[34] S. Guruswamy, A. LeClair and A. W. W. Ludwig, $g l(N \mid N)$ super-current algebras for disordered Dirac fermions in two dimensions, Nucl. Phys. B583 (2000) 475-512 cond-mat/9909143.

[35] A. W. W. Ludwig, A free field representation of the osp $(2 \mid 2)$ current algebra at level $k=-2$, and Dirac fermions in a random $S U(2)$ gauge potential, cond-mat/0012189.

[36] V. Schomerus and H. Saleur, The GL(1|1) WZW model: From supergeometry to logarithmic CFT, Nucl. Phys. B734 (2006) 221-245 hep-th/0510032.

[37] H. Saleur and V. Schomerus, On the $S U(2 \mid 1)$ WZW model and its statistical mechanics applications, hep-th/0611147. To appear in Nucl. Phys. B.

[38] B. L. Feigin and E. V. Frenkel, A family of representations of affine Lie algebras, Uspekhi Mat. Nauk 43 (1988) 227-228.

[39] A. Gerasimov, A. Morozov, M. Olshanetsky, A. Marshakov and S. L. Shatashvili, Wess-Zumino-Witten model as a theory of free fields, Int. J. Mod. Phys. A5 (1990) $2495-2589$.

[40] P. Bouwknegt, J. G. McCarthy and K. Pilch, Free field realizations of WZNW models: BRST complex and its quantum group structure, Phys. Lett. B234 (1990) 297-303.

[41] B. L. Feigin and E. V. Frenkel, Representations of affine Kac-Moody algebras, bosonization and resolutions, Lett. Math. Phys. 19 (1990) 307-317.

[42] J. Rasmussen, Free field realizations of affine current superalgebras, screening currents and primary fields, Nucl. Phys. B510 (1998) 688-720 hep-th/9706091.

[43] K. Ito, J. O. Madsen and J. L. Petersen, Free field representations of extended superconformal algebras, Nucl. Phys. B398 (1993) 425-458 [hep-th/9207009. 
[44] K. Ito, J. O. Madsen and J. L. Petersen, Extended superconformal algebras from classical and quantum Hamiltonian reduction, in Proceedings of the International Workshop on String Theory, Quantum Gravity and the Unification of Fundamental Interactions, Rome, pp. 302-330, 1992. hep-th/9211019.

[45] V. G. Kac, Lie superalgebras, Adv. Math. 26 (1977) 8-96.

[46] C. R. Nappi and E. Witten, A WZW model based on a nonsemisimple group, Phys. Rev. Lett. 71 (1993) 3751-3753 hep-th/9310112.

[47] G. D'Appollonio and E. Kiritsis, String interactions in gravitational wave backgrounds, Nucl. Phys. B674 (2003) 80-170 [hep-th/0305081.

[48] Y. M. Zou, Categories of finite-dimensional weight modules over type I classical Lie superalgebras, J. Algebra 180 (1996) 459-482.

[49] J. Brundan, Tilting modules for Lie superalgebras, Comm. Algebra 32 (2004) 2251-2268 math.RT/0209235.

[50] V. Kac, Representations of classical Lie superalgebras, in Differential geometrical methods in mathematical physics, II (Proc. Conf., Univ. Bonn, Bonn, 1977), vol. 676 of Lecture Notes in Math., pp. 597-626. Springer, Berlin, 1978.

[51] V. Serganova, Characters of irreducible representations of simple Lie superalgebras, in Proceedings of the International Congress of Mathematicians, Vol. II (Berlin, 1998), no. Extra Vol. II, pp. 583-593 (electronic), 1998.

[52] J. Germoni, Indecomposable representations of special linear Lie superalgebras, J. Algebra 209 (1998) 367-401.

[53] J. Germoni, Indecomposable representations of osp $(3 \mid 2), D(2,1 ; \alpha)$ and $G(3)$, Bol. Acad. Nac. Cienc. (Córdoba) 65 (2000) 147-163. Colloquium on Homology and Representation Theory (Spanish) (Vaquerías, 1998).

[54] S. J. Gates, M. T. Grisaru, M. Rocek and W. Siegel, Superspace, or one thousand and one lessons in supersymmetry, Front. Phys. 58 (1983) 1-548 hep-th/0108200.

[55] A. M. Polyakov and P. B. Wiegmann, Goldstone fields in two-dimensions with multivalued actions, Phys. Lett. B141 (1984) 223-228.

[56] H. Sugawara, A field theory of currents, Phys. Rev. 170 (1968) 1659-1662.

[57] D. Gepner and E. Witten, String theory on group manifolds, Nucl. Phys. B278 (1986) 493.

[58] A. Hüffmann, On representations of supercoalgebras, J. Phys. A27 (1994) 6421-6432 hep-th/9403100.

[59] M. Scheunert and R. B. Zhang, The general linear supergroup and its Hopf superalgebra of regular functions, J. Algebra 254 (2002), no. 1 44-83.

[60] V. G. Kac and D. H. Peterson, Infinite dimensional Lie algebras, theta functions and modular forms, Adv. Math. 53 (1984) 125-264. 
[61] V. G. Kac, Infinite dimensional Lie algebras. Cambridge University Press, Cambridge, 3rd ed., 1990.

[62] J. Brundan, Kazhdan-Lusztig polynomials and character formulae for the Lie superalgebra $g l(m \mid n)$, J. Amer. Math. Soc. 16 (2003) 185-231 (electronic) math.RT/0203011.

[63] V. G. Kac and M. Wakimoto, Integrable highest weight modules over affine superalgebras and number theory, in Lie theory and geometry, vol. 123 of Progr. Math., pp. 415-456.

Birkhäuser Boston, Boston, MA, 1994. hep-th/9407057.

[64] V. G. Kac and M. Wakimoto, Integrable heighest weights modules over affine superalgebras and Appell's function, Comm. Math. Phys. 215 (2001) 631-682 math-ph/0006007.

[65] M. Kreuzer and A. N. Schellekens, Simple currents versus orbifolds with discrete torsion: A complete classification, Nucl. Phys. B411 (1994) 97-121 hep-th/9306145.

[66] J. Fuchs, I. Runkel and C. Schweigert, TFT construction of RCFT correlators. III: Simple currents, Nucl. Phys. B694 (2004) 277-353 hep-th/0403157.

[67] A. Rogers, Super Lie groups: Global topology and local structure, J. Math. Phys. 22 (1981) 939.

[68] H. G. Kausch, Extended conformal algebras generated by a multiplet of primary fields, Phys. Lett. B259 (1991) 448-455.

[69] M. R. Gaberdiel and H. G. Kausch, A rational logarithmic conformal field theory, Phys. Lett. B386 (1996) 131-137 hep-th/9606050.

[70] B. L. Feigin, A. M. Gainutdinov, A. M. Semikhatov and I. Y. Tipunin, Modular group representations and fusion in logarithmic conformal field theories and in the quantum group center, Commun. Math. Phys. 265 (2006) 47-93 hep-th/0504093.

[71] G. Götz, T. Quella and V. Schomerus, Representation theory of sl(2|1), J. Algebra 312 (2007) 829-848 hep-th/0504234.

[72] K. Erdmann, E. Green, N. Snashall and R. Taillefer, Representation theory of the Drinfel'd doubles of a family of Hopf algebras, Journal of Pure and Applied Algebra 204 (2006) 413-454 math.RT/0410017.

[73] B. L. Feigin, A. M. Gainutdinov, A. M. Semikhatov and I. Y. Tipunin, Kazhdan-Lusztig correspondence for the representation category of the triplet $W$-algebra in logarithmic conformal field theory, Theor. Math. Phys. 148 (2006) 1210-1235 math.qa/0512621.

[74] H. Eberle and M. Flohr, Virasoro representations and fusion for general augmented minimal models, J. Phys. A39 (2006) 15245-15286 hep-th/0604097.

[75] B. L. Feigin, A. M. Gainutdinov, A. M. Semikhatov and I. Y. Tipunin, Logarithmic extensions of minimal models: Characters and modular transformations, Nucl. Phys. B757 (2006) 303-343 hep-th/0606196.

[76] G. Götz, T. Quella and V. Schomerus, Tensor products of psl $(2 \mid 2)$ representations, hep-th/0506072. 
[77] B. L. Feigin, A. M. Gainutdinov, A. M. Semikhatov and I. Y. Tipunin, Kazhdan-Lusztig-dual quantum group for logarithmic extensions of Virasoro minimal models, math.qa/0606506.

[78] T. Creutzig, T. Quella and V. Schomerus, "D-branes on GL(1|1)." Work in progress.

[79] K. Gawedzki and A. Kupiainen, G/H conformal field theory from gauged WZW model, Phys. Lett. B215 (1988) 119-123.

[80] K. Gawedzki and A. Kupiainen, Coset construction from functional integrals, Nucl. Phys. B320 (1989) 625.

[81] D. Karabali, Q.-H. Park, H. J. Schnitzer and Z. Yang, A GKO construction based on a path integral formulation of gauged Wess-Zumino-Witten actions, Phys. Lett. B216 (1989) 307.

[82] D. Karabali and H. J. Schnitzer, BRST quantization of the gauged WZW action and coset conformal field theories, Nucl. Phys. B329 (1990) 649.

[83] R. Zhang and Y. Zou, Spherical functions on homogeneous superspaces, J. Math. Phys. 46 (2005) 043513 math.RT/0608693.

[84] N. Berkovits, C. Vafa and E. Witten, Conformal field theory of AdS background with Ramond-Ramond flux, JHEP 03 (1999) 018 hep-th/9902098.

[85] N. Berkovits, Super-Poincaré covariant quantization of the superstring, JHEP 04 (2000) 018 hep-th/0001035.

[86] P. A. Grassi, G. Policastro, M. Porrati and P. Van Nieuwenhuizen, Covariant quantization of superstrings without pure spinor constraints, JHEP 10 (2002) 054 hep-th/0112162.

[87] P. A. Grassi, G. Policastro and P. van Nieuwenhuizen, The quantum superstring as a WZNW model, Nucl. Phys. B676 (2004) 43-63 hep-th/0307056.

[88] J. L. Jacobsen and H. Saleur, The arboreal gas and the supersphere sigma model, Nucl. Phys. B716 (2005) 439-461 cond-mat/0502052. 